Article

\title{
Syntheses of 1-Aryl-5-nitro-1H-indazoles and a General One-Pot Route to 1-Aryl-1H-indazoles
}

\author{
Joel K. Annor-Gyamfi, Krishna Kumar Gnanasekaran and Richard A. Bunce * \\ Department of Chemistry, Oklahoma State University, Stillwater, OK 74078-3071, USA; \\ jannorg@okstate.edu (J.K.A.-G.); krishna.gnanasekaran@okstate.edu (K.K.G.) \\ * Correspondence: rab@okstate.edu; Tel.: +1-405-744-5952; Fax: +1-405-744-6007
}

Received: 23 February 2018; Accepted: 14 March 2018; Published: 16 March 2018

\begin{abstract}
An efficient route to substituted 1-aryl-1H-indazoles has been developed and optimized. The method involved the preparation of arylhydrazones from acetophenone or benzaldehyde substituted by fluorine at $\mathrm{C} 2$ and nitro at $\mathrm{C} 5$, followed by deprotonation and nucleophilic aromatic substitution $\left(\mathrm{S}_{\mathrm{N}} \mathrm{Ar}\right)$ ring closure in $45-90 \%$. Modification of this procedure to a one-pot domino process was successful in the acetophenone series (73-96\%), while the benzaldehyde series (63-73\%) required a step-wise addition of reagents. A general one-pot protocol for 1-aryl-1H-indazole formation without the limiting substitution patterns required for the $\mathrm{S}_{\mathrm{N}} \mathrm{Ar}$ cyclization has also been achieved in $62-78 \%$ yields. A selection of 1-aryl- $1 H$-indazoles was prepared in high yield by a procedure that requires only a single laboratory operation.
\end{abstract}

Keywords: 1-aryl-5-nitro-1H-indazole; 1 -aryl- $1 H$-indazole; arylhydrazone; $\mathrm{S}_{\mathrm{N}} \mathrm{Ar}$ reaction; Ullmann reaction

\section{Introduction}

Our synthetic methods program has recently been focused on the use of nucleophilic aromatic substitution $\left(\mathrm{S}_{\mathrm{N}} \mathrm{Ar}\right)$-terminated domino reactions as a means to prepare heterocyclic compounds [1-4]. The current project has developed a synthesis of 1-aryl- $1 H$-indazoles, which are core ring structures in a broad range of biologically active compounds (Figure 1). Among the compounds pictured, benzydamine (1) is a well-known NSAID drug [5], while alcohol 2 has likewise shown promise as an anti-inflammatory agent [6]. The urea-based structure 3 has recently been judged to possess significant tumor antiproliferative properties [7]. Similarly, amide 4 has also demonstrated anticancer activity $[8,9]$.

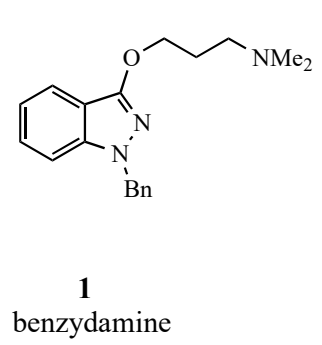

anti-inflammatory agents

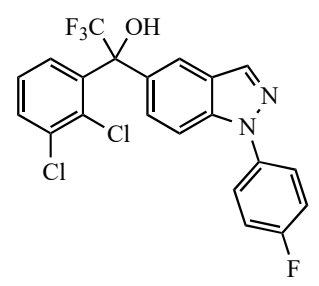

2

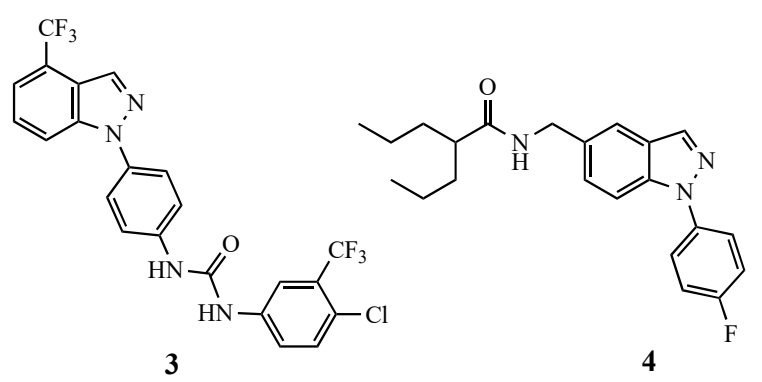

anticancer agents

Figure 1. Drugs incorporating 1-aryl-1H-indazoles.

Several strategies have previously been disclosed as routes to 1 -aryl- $1 H$-indazoles. All but one required multiple steps. The closest work to that presented here utilized a two-step sequence involving 
acid catalyzed formation of the arylhydrazone, followed by a copper(I)-catalyzed Ullmann-type coupling to close the ring [10]. A related investigation described a similar cyclization of arylhydrazones from 2-(2-haloaryl)-2-oxoacetic esters [11]. A complementary approach utilized a copper(I)-promoted coupling of $N$-aroyl- $N^{\prime}$-arylhydrazines with 2-bromoaryl ketones or aldehydes [12], followed by deacylation and condensative ring closure. Two additional accounts examined (1) an $\mathrm{S}_{\mathrm{N}} \mathrm{Ar}$ displacement of nitro by anions derived from arylhydrazones of 2-nitrobenzaldehydes [13] and (2) a one-step conversion from salicylaldehydes and arylhydrazine hydrochlorides [14]. Other reports have described a one-pot condensation- $\mathrm{S}_{\mathrm{N}} \mathrm{Ar}$ protocol using 2-fluorobenzaldehyde to prepare $1 H$-indazoles, but these utilized more reactive hydrazines and did not yield 1-aryl-substituted derivatives $[15,16]$. Along a different line, another study delineated a novel [3+2]-type annulation between arynes and arylhydrazones in the presence of fluoride under aerobic conditions [17]. Finally, syntheses promoted by palladium- [18] and rhodium-based [19] catalysts have also furnished these targets. A full listing of methods to prepare $1 \mathrm{H}$-indazoles, with and without the $\mathrm{N} 1$ aryl group, have been cataloged in three published reviews [20-22].

The number of steps and the waste generated are two important considerations in planning synthetic procedures. Our aim, in the current study, was to develop a one-pot domino or consecutive process [23-25] to accomplish the synthesis of 1-aryl-1H-indazoles. Utilizing these techniques, a single operation-one set-up and one work-up—would generate members of this emerging class of heterocyclic building blocks rapidly and with a minimum of chemical waste. The details of our efforts towards this goal are given below.

\section{Results and Discussion}

A logical route to $1 \mathrm{H}$-indazoles would involve the ring closure of an arylhydrazone anion derived from an appropriately substituted aromatic ketone or aldehyde. Although the arylhydrazones would form as two isomers, with the $E$ isomer (less favorable for cyclization) predominating, these should readily interconvert with heat [26] and allow access to the $Z$ isomer required for ring closure. Furthermore, the pKa of the phenylhydrazone proton $(\mathrm{Ar}=\mathrm{Ph})$ is 21.5 [27], and with slight variations due to substituents on the aromatic ring, should permit the use of $\mathrm{K}_{2} \mathrm{CO}_{3}$ in a polar aprotic solvent as the deprotonating agent $[28,29]$. Optimally, these annulations would proceed in one pot, but we initially sought to determine the feasibility of the sequence by evaluating each transformation separately. Once each step was optimized, attempts would then be made to merge the two reactions into a single laboratory operation. Finally, since this would generate structures with limited substitution patterns, we also aspired to generalize the process to include structures without $S_{N} A r$ activating groups.

Although there are numerous examples of $S_{N}$ Ar-type reactions to generate five-membered rings fused to a benzene, some strain would be expected in ring formations involving closure of a fragment incorporating three $\mathrm{sp}^{2}$ atoms onto an aromatic nucleus [30,31]. Thus, $2^{\prime}$-fluoro-5'-nitroacetophenone (5) and 2-fluoro-5-nitrobenzaldehyde (6) were initially reacted with hydrazine hydrate $\left(\mathrm{NH}_{2} \mathrm{NH}_{2} \cdot \mathrm{H}_{2} \mathrm{O}\right)$ to assess the feasibility of forming indazoles in this manner. Due to the alpha-effect [32], hydrazine is an exceptionally potent nucleophile and might be expected to react without added base. Indeed, treatment of $\mathbf{5}$ and $\mathbf{6}$ with $\mathrm{NH}_{2} \mathrm{NH}_{2} \cdot \mathrm{H}_{2} \mathrm{O}$ (3.0 equivalents for $\mathbf{5}$ and 2.0 equivalents for $\mathbf{6}$ ) in $\mathrm{N}, \mathrm{N}$-dimethylformamide (DMF) at $23{ }^{\circ} \mathrm{C}$ for two hours resulted in high yields of targets 7 and 8, respectively (see Scheme 1). Encouraged by this result, we forged ahead to prepare 1-aryl-5-nitro-1H-indazoles. 
<smiles>[R]C(=O)c1cc([N+](=O)[O-])ccc1F</smiles>

$5(\mathrm{R}=\mathrm{Me})$

$6(\mathrm{R}=\mathrm{H})$

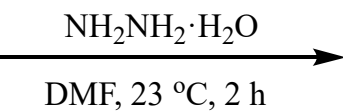

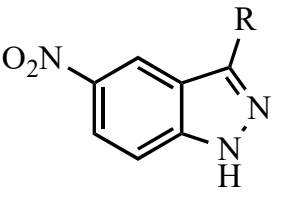

$7(\mathrm{R}=\mathrm{Me}) 98 \%$

$8(\mathrm{R}=\mathrm{H}) \quad 90 \%$

Scheme 1. Reaction of 5 and 6 with hydrazine hydrate.

Formation of the intermediate arylhydrazones $\mathbf{9}$ and $\mathbf{1 0}$ was initially addressed. This involved warming a DMF solution of the carbonyl compound with an arylhydrazine hydrochloride $\left(\mathrm{ArNHNH} \mathrm{H}_{2} \cdot \mathrm{HCl}\right.$ ) at $50{ }^{\circ} \mathrm{C}$ for two to three hours. DMF was used as a solvent for this process since we anticipated that a one-pot procedure where the hydrazone was prepared, deprotonated, and cyclized would likely require the use of a polar aprotic solvent. Optimization studies indicated that DMF was a suitable medium and the use of 3.0 equivalents of $\mathrm{ArNHNH}_{2} \cdot \mathrm{HCl}$ with acetophenone 5 and 2.0 equivalents with benzaldehyde 6 afforded the highest yields of 9 and $\mathbf{1 0}$. In all cases, the intermediate hydrazones were isolated in $\geq 70 \%$ yield from $\mathbf{5}$ and $\geq 50 \%$ yield from $\mathbf{6}$. Interestingly, compounds arising from direct $\mathrm{S}_{\mathrm{N}} \mathrm{Ar}$ displacement of fluoride by the arylhydrazine were not observed to any significant extent under these conditions. While the product of addition-elimination by hydrazine is known to cyclize to a $1 \mathrm{H}$-indazole [33,34], ring closure of the analogous product from arylhydrazine would give a 2-aryl-2H-indazole. Neither the arylhydrazine $\mathrm{S}_{\mathrm{N}} \mathrm{Ar}$ product nor the $2 \mathrm{H}$-indazole were detected.

Cyclization of hydrazones 9 and $\mathbf{1 0}$ required base, higher temperature and occasionally extended reaction times. This reaction was performed in DMF at $90{ }^{\circ} \mathrm{C}$ and found to work best using 3.0 equivalents of $\mathrm{K}_{2} \mathrm{CO}_{3}$. Yields were slightly lower from the benzaldehyde since this substrate led to a product that was unhindered at $\mathrm{C} 3$ and possibly susceptible to nucleophilic attack by various species in the reaction. A summary of optimization experiments is given in the Electronic Supplemental Inofrmation, and the results of our two-step synthesis of 1-aryl-5-nitro- $1 \mathrm{H}$-indazoles are summarized in Table 1.

Modification of the protocol to allow for a one-pot procedure had to overcome several problems that could lead to unwanted impurities. Since water is produced during arylhydrazone formation, some conversion of the $\mathrm{K}_{2} \mathrm{CO}_{3}$ to $\mathrm{KOH}$ would be expected. Furthermore, $\mathrm{KOH}$ could hydrolyze the DMF solvent to form $\mathrm{Me}_{2} \mathrm{NH}$. Both of these nucleophiles $\left(\mathrm{OH}^{-}\right.$and $\left.\mathrm{Me}_{2} \mathrm{NH}\right)$ could potentially undergo competitive $\mathrm{S}_{\mathrm{N}} \mathrm{Ar}$ addition to the carbonyl containing substrate. While no significant product resulting from $\mathrm{OH}^{-}$addition was observed, some of the $\mathrm{Me}_{2} \mathrm{NH}$ addition product was noted when prolonged heating was required. These side reactions were suppressed by adding $30 \mathrm{wt} \%$ (relative to 5 or 6) of powdered $4 \AA$ A molecular sieves to scavenge water produced during the initial condensation and using anhydrous 1,3-dimethyl-3,4,5,6-tetrahydro-2(1H)-pyrimidinone (DMPU) as the solvent to minimize hydrolysis.

The one-step domino process was successful for the conversion of ketone 5 to indazoles 11, and in most cases, the yield was superior to the two-step sequence (see Table 2). Reactions of aldehyde 6 to give 12, however, were more limited and several of the substrates bearing acidic functions on the aromatic ring failed to react. For aldehydes that did generate a product, sequential treatment with $\mathrm{ArNHNH}_{2} \cdot \mathrm{HCl}$ and base afforded yields that also surpassed the two-step process. In both one-pot procedures, it was often noted that the cyclization required a longer reaction time when the aryl group of the hydrazone was substituted by an electron-withdrawing group. This would be expected since the arylhydrazone anion would be less nucleophilic with a stabilizing group at $\mathrm{C} 2$ or $\mathrm{C} 4$ of the hydrazone aromatic ring [35]. Conversely, this same anion with an electron-donating substituent at C2 or C4 should be more reactive with potentially shorter reaction times. 
Table 1. Yields for the two-step synthesis of 1-aryl-5-nitro- $1 H$-indazoles.

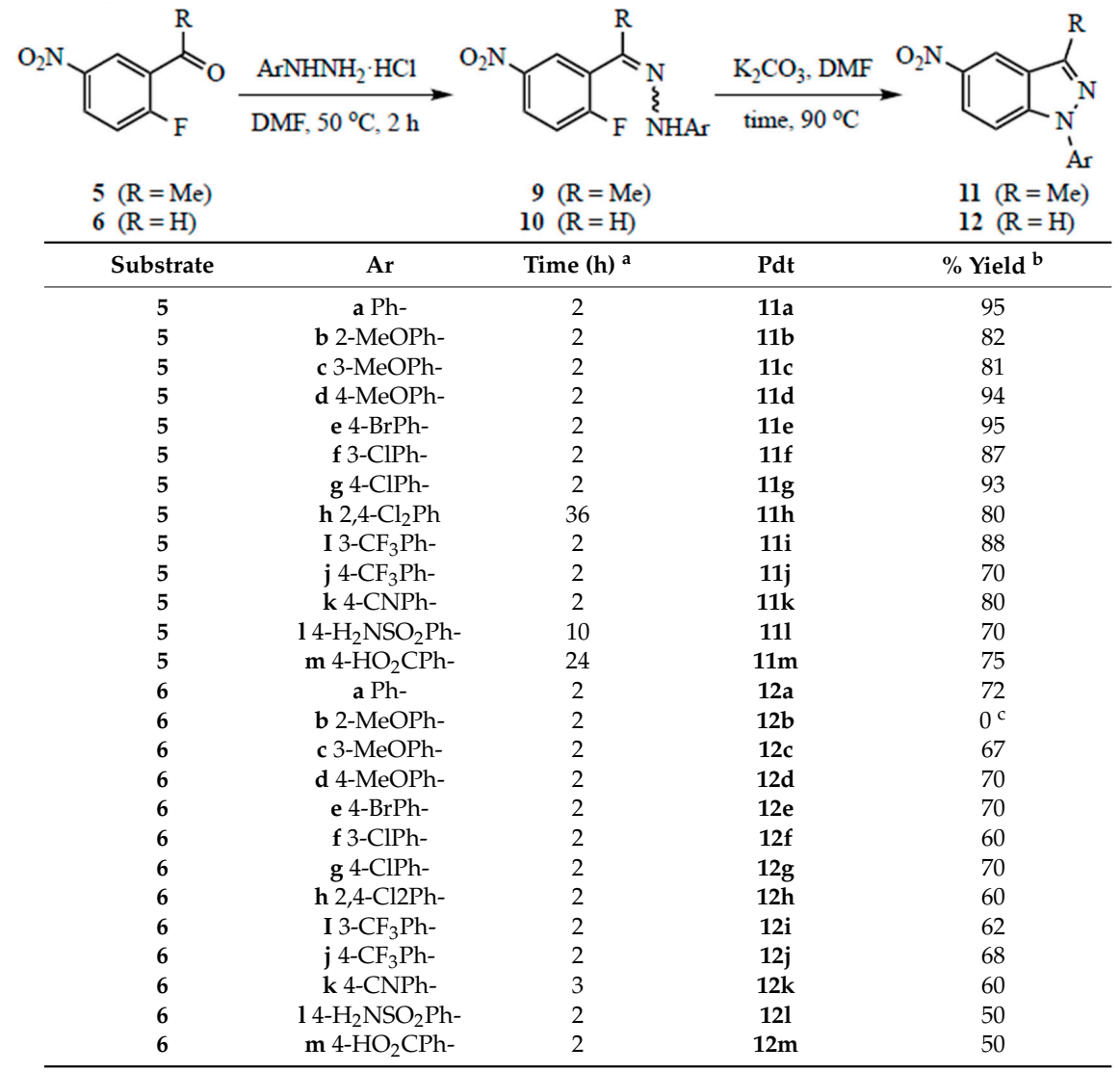

a All hydrazones were generated in two hours. Times given are for the final cyclization. ${ }^{\mathrm{b}}$ Isolated yield of $1 \mathrm{H}$-indazole for the two-step sequence. ${ }^{\mathrm{c}}$ Only the hydrazone was isolated.

Table 2. Yields for the modified one-pot synthesis of 1-aryl-5-nitro- $1 H$-indazoles.

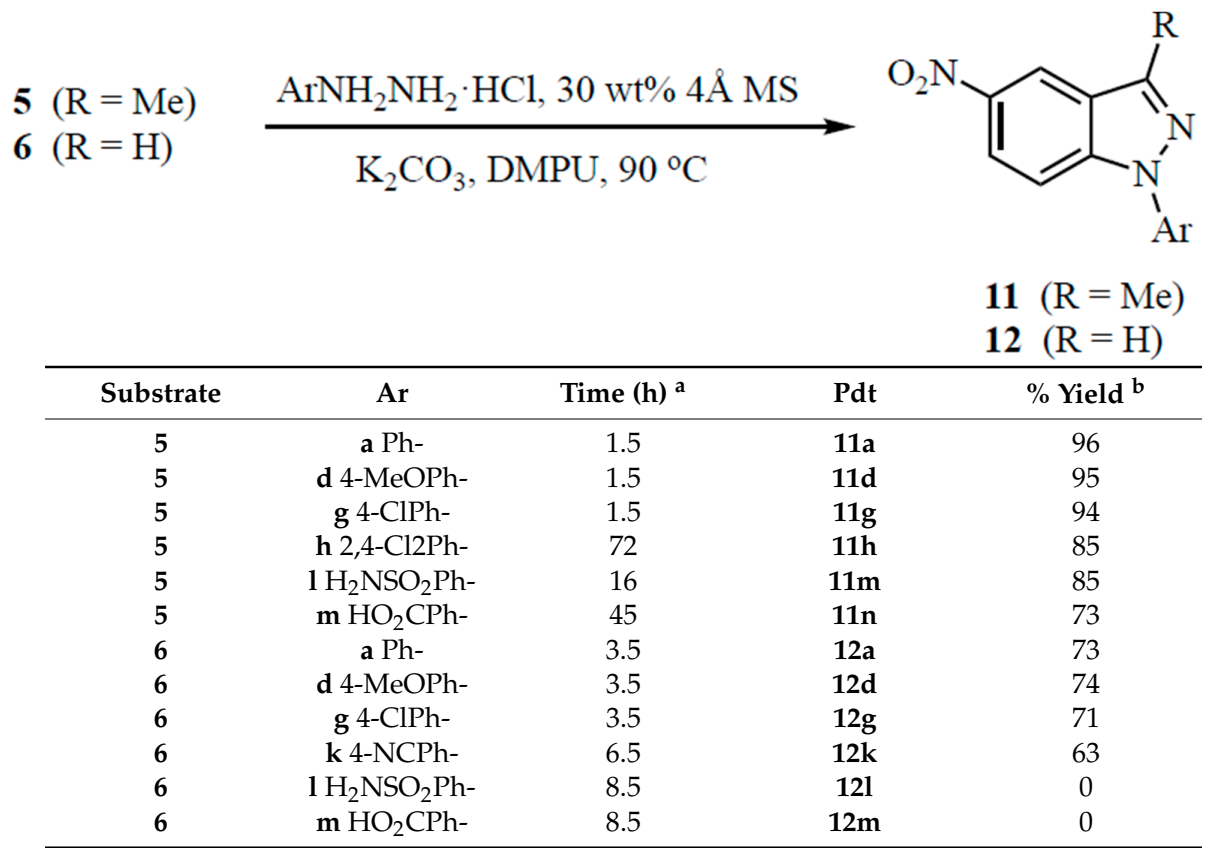

a For 5 , time is the total reaction time. For 6 , the hydrazone was allowed to form for $1.5 \mathrm{~h}$ at $90{ }^{\circ} \mathrm{C}$ before base was added; time is for the final cyclization. ${ }^{\mathrm{b}}$ Isolated yield. DMPU: 1,3-dimethyl-3,4,5,6-tetrahydro-2(1H)-pyrimidinone. 
Finally, we sought to develop a general approach to $1 H$-indazoles unrestricted by the substitution patterns needed for the $S_{N} A r$ ring closure (see Table 3). Protocol development efforts indicated that this variant of the reaction required sequential addition of the reagents. The hydrazone was initially generated using 1.5 equivalents of the $\mathrm{ArNHNH}_{2} \cdot \mathrm{HCl}$ and then cyclized by addition of $20 \mathrm{~mol} \%$ of copper(I) iodide (CuI) and 2.5 equivalents of $\mathrm{K}_{2} \mathrm{CO}_{3}$, all in the same flask at $90{ }^{\circ} \mathrm{C}$. Again, the key to success in this transformation was the inclusion of $30 \mathrm{wt} \%$ of powdered $4 \AA$ molecular sieves and the use of DMPU as the solvent. Without activating groups on the ring, Ullmann conditions ( $\mathrm{CuI}$ and base) for the final ring closure were essential. An earlier paper noted that this copper(I)-catalyzed ring closure was facilitated by the addition of $20 \mathrm{~mol} \%$ of L-proline or 4-hydroxy-L-proline [8,9]. In the current procedure, however, addition of these ligands had no impact on the yield of the reaction. The cyclization using 2'-bromoacetophenone (13), 2-bromobenzaldehyde (14) and 2-chloronicotinaldehyde (15) was performed using phenylhydrazine as well as electron-rich and electron-poor arylhydrazines in relatively uniform yields. Though it was expected that the nicotinaldehyde derivatives would not need a copper catalyst, since it could directly cyclize by a $S_{N} A r$ reaction, this ring closure failed to proceed to completion without this additive. Overall, our procedure permits the rapid construction of 1-aryl- $1 H$-indazoles in a single reaction vessel without isolation or purification of intermediates.

Table 3. Yields for the general one-pot synthesis of 1-aryl-1H-indazoles.

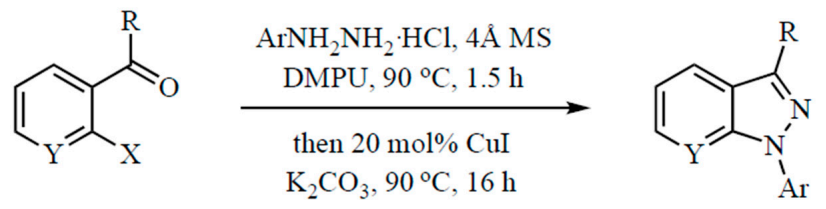

\begin{tabular}{|c|c|c|c|}
\hline \multicolumn{2}{|c|}{$\begin{array}{l}13(\mathrm{X}=\mathrm{Br}, \mathrm{Y}=\mathrm{CH} ; \mathrm{R}=\mathrm{Me}) \\
14(\mathrm{X}=\mathrm{Br}, \mathrm{Y}=\mathrm{CH} ; \mathrm{R}=\mathrm{H}) \\
15(\mathrm{X}=\mathrm{Cl}, \mathrm{Y}=\mathrm{N} ; \mathrm{R}=\mathrm{H})\end{array}$} & \multicolumn{2}{|c|}{$\begin{array}{l}16(\mathrm{Y}=\mathrm{CH} ; \mathrm{R}=\mathrm{Me}) \\
17(\mathrm{Y}=\mathrm{CH} ; \mathrm{R}=\mathrm{H}) \\
18(\mathrm{Y}=\mathrm{N} ; \mathrm{R}=\mathrm{H})\end{array}$} \\
\hline Substrate & Ar & Product & $\%$ Yield $^{a}$ \\
\hline 13 & a Ph- & $16 a$ & 81 \\
\hline 13 & d 4-MeOPh- & $16 d$ & 83 \\
\hline 13 & k 4-CNPh- & 161 & 87 \\
\hline 14 & a Ph- & $17 a$ & 77 \\
\hline 14 & d 4-MeOPh- & $17 d$ & 72 \\
\hline 14 & k 4-CNPh- & 171 & 79 \\
\hline 15 & a Ph- & $18 a$ & 68 \\
\hline 15 & d 4-MeOPh- & $18 d$ & 62 \\
\hline 15 & k 4-CNPh- & 181 & 78 \\
\hline
\end{tabular}

a Isolated yield.

\section{Experimental Section}

\subsection{General Methods}

Unless otherwise indicated, all reactions were carried out under dry $\mathrm{N}_{2}$ in oven-dried glassware. All reagents and solvents were used as received. Reactions were monitored by thin layer chromatography on silica gel GF plates (Analtech No. 21521, Newark, DE, USA). Preparative separations were performed by flash chromatography on silica gel (Davisil ${ }^{\circledR}$, grade 62, 60-200 mesh, Sorbent Technologies, Norcross, GA, USA) containing UV-active phosphor (Sorbent Technologies No. UV-05) slurry packed into quartz columns. Band elution for all chromatographic separations was monitored using a hand-held UV lamp (Fisher Scientific, Pittsburgh, PA, USA). Melting points were obtained using a MEL-TEMP apparatus (Laboratory Devices, Cambridge, MA, USA) and are uncorrected. FT-IR spectra were run using a Varian Scimitar FTS 800 spectrophotometer (Varian Instruments, Randolph, MA, USA) as thin films or nujol mulls on $\mathrm{NaCl}$ disks. ${ }^{1} \mathrm{H}-$ and ${ }^{13} \mathrm{C}-\mathrm{NMR}$ spectra were measured using a Bruker Avance 400 system (Bruker Corporation, Billerica, MA, USA) 
in the indicated solvents at $400 \mathrm{MHz}$ and $101 \mathrm{MHz}$, respectively, with $\left(\mathrm{CH}_{3}\right)_{4} \mathrm{Si}$ as the internal standard; coupling constants $(J)$ are given in Hz. Unit resolution mass spectra were obtained using a Shimadzu LCMS-2010EV system (Shimadzu Corporation, Columbia, MD, USA). Elemental analyses $( \pm 0.4 \%)$ were determined by Atlantic Microlabs (Norcross, GA, USA).

\subsection{Indazoles from 2'-Fluoro-5'-nitroacetophenone (5) or 2-Fluoro-5-nitrobenzaldehyde (6) with $\mathrm{NH}_{2} \mathrm{NH}_{2} \cdot \mathrm{H}_{2} \mathrm{O}$}

To a stirred solution of 5 or $6(1.0 \mathrm{mmol})$ in DMF $(5 \mathrm{~mL})$ at $23{ }^{\circ} \mathrm{C}$ was added $\mathrm{NH}_{2} \mathrm{NH}_{2} \cdot \mathrm{H}_{2} \mathrm{O}$ (3.0 mmol for 5, $2.0 \mathrm{mmol}$ for 6). The solution was stirred at $23{ }^{\circ} \mathrm{C}$ for $2 \mathrm{~h}$ at which time thin layer chromatography (TLC, 20\% EtOAc in hexanes) indicated complete consumption of the starting carbonyl compound. The crude mixture was added to water and extracted with EtOAc $(2 \times 15 \mathrm{~mL})$. The combined organic layers were washed with water and saturated aq $\mathrm{NaCl}$, dried $\left(\mathrm{MgSO}_{4}\right)$, filtered, and concentrated under vacuum to give the pure indazole products.

3-Methyl-5-nitro-1H-indazole (7). Yield: $173 \mathrm{mg}(0.98 \mathrm{mmol}, 98 \%)$ as a yellow solid, m.p. $213-214{ }^{\circ} \mathrm{C}$; IR: 1517, $1332 \mathrm{~cm}^{-1} ;{ }^{1} \mathrm{H}-\mathrm{NMR}$ (DMSO- $\left.d_{6}\right): \delta 13.1$ (br s, $\left.1 \mathrm{H}\right), 8.79(\mathrm{~s}, 1 \mathrm{H}), 8.18(\mathrm{~d}, J=9.1 \mathrm{~Hz}, 1 \mathrm{H})$, $7.64(\mathrm{~d}, J=9.1 \mathrm{~Hz}, 1 \mathrm{H}), 2.59(\mathrm{~s}, 3 \mathrm{H}) ;{ }^{13} \mathrm{C}-\mathrm{NMR}\left(\mathrm{DMSO}-d_{6}\right): \delta 145.4,143.1,141.3,121.9,121.3,118.8$, 111.2, 12.1; MS: $m / z 177\left(\mathrm{M}^{+}\right)$, calculated $m / z$ 177.05. Anal. Calcd. for $\mathrm{C}_{8} \mathrm{H}_{7} \mathrm{~N}_{3} \mathrm{O}_{2}: \mathrm{C}, 54.24 ; \mathrm{H}, 3.98$; $\mathrm{N}, 23.72$. Found: C, 54.29; H, 4.01; N, 23.59 .

5-Nitro-1H-indazole (8). Yield: $147 \mathrm{mg}(0.90 \mathrm{mmol}, 90 \%)$ as a tan solid, m.p. 207-208 ${ }^{\circ} \mathrm{C}$; IR: 1534, $1341 \mathrm{~cm}^{-1} ;{ }^{1} \mathrm{H}-\mathrm{NMR}$ (DMSO- $\left.d_{6}\right): \delta 13.7$ (br s, $\left.1 \mathrm{H}\right), 8.84(\mathrm{~s}, 1 \mathrm{H}), 8.41(\mathrm{~s}, 1 \mathrm{H}), 8.19(\mathrm{~d}, J=9.0 \mathrm{~Hz}$, $1 \mathrm{H}), 7.74(\mathrm{~d}, J=9.0 \mathrm{~Hz}, 1 \mathrm{H}) ;{ }^{13} \mathrm{C}-\mathrm{NMR}\left(\mathrm{DMSO}-d_{6}\right): \delta 142.3,142.0,137.2,122.5,121.3,119.3,111.5$; MS: $m / z 163\left(\mathrm{M}^{+}\right)$, calculated $m / z$ 163.04. Anal. Calcd. for $\mathrm{C}_{7} \mathrm{H}_{5} \mathrm{~N}_{3} \mathrm{O}_{2}: \mathrm{C}, 51.54 ; \mathrm{H}, 3.09 ; \mathrm{N}, 25.76$. Found: C, 51.85; H, 3.15; N, 25.54.

\subsection{Indazoles from $\mathbf{5}$ or $\mathbf{6}$ with $\mathrm{ArNHNH} \mathrm{H}_{2} \cdot \mathrm{HCl}$. Method A. Representative Two-Step Procedure}

To a stirred solution of the carbonyl compound $(1.0 \mathrm{mmol})$ in DMF $(5 \mathrm{~mL})$ at $50{ }^{\circ} \mathrm{C}$ (oil bath) was added $\mathrm{ArNHNH}_{2} \cdot \mathrm{HCl}(3.0 \mathrm{mmol}$ for 5, $2.0 \mathrm{mmol}$ for 6$)$ and the solution was stirred until TLC ( $20 \%$ EtOAc in hexanes) indicated complete conversion (roughly $2 \mathrm{~h}$ ). The crude reaction mixture was added to water and extracted with EtOAc $(2 \times 15 \mathrm{~mL})$. The combined organic layers were washed with water and saturated aq $\mathrm{NaCl}$, dried $\left(\mathrm{MgSO}_{4}\right)$, filtered, and concentrated under vacuum to give the crude hydrazones. The crude products were stirred with $20 \%$ ether/pentane for $1 \mathrm{~h}$, filtered and dried to give arylhydrazones 9 and 10. Characterization data for these materials are given in the ESI.

To a stirred solution of the arylhydrazone $(1.00 \mathrm{mmol})$ in DMF $(5 \mathrm{~mL})$ at $90{ }^{\circ} \mathrm{C}$ (oil bath) was added anhydrous $\mathrm{K}_{2} \mathrm{CO}_{3}(3.0 \mathrm{mmol})$ and the mixture was stirred for the time indicated in Table 1 . The reaction mixture was added to water, extracted with EtOAc, dried $\left(\mathrm{MgSO}_{4}\right)$, filtered, and concentrated under vacuum to afford the crude product. Indazoles from 5 were stirred with $20 \%$ ether in pentane, filtered and dried, while those from 6 were purified by silica gel chromatography on a $20 \mathrm{~cm} \times 2 \mathrm{~cm}$ column eluted with increasing concentrations of EtOAc in hexanes. Yields as well as physical and spectral data are given below.

\subsection{Indazoles from $\mathbf{5}$ or $\mathbf{6}$ with $\mathrm{ArNHNH} \mathrm{H}_{2} \cdot \mathrm{HCl}$. Method B. Representative One-Pot Procedure}

To a stirred solution of the carbonyl com.p.ound $(1.0 \mathrm{mmol})$ in DMPU $(5 \mathrm{~mL})$ were added powdered $4 \AA$ molecular sieves ( $30 \mathrm{wt} \%$ relative to the carbonyl substrate), $\mathrm{ArNHNH} \cdot \mathrm{HCl}(3.0 \mathrm{mmol}$ for 5, $2.0 \mathrm{mmol}$ for 6), and $\mathrm{K}_{2} \mathrm{CO}_{3}(3.0 \mathrm{mmol}$ for 5, $2.0 \mathrm{mmol}$ for 6). For 5, all reagents were placed in the flask and heated to $90{ }^{\circ} \mathrm{C}$; for 6 , the hydrazone was allowed to form at $90^{\circ} \mathrm{C}(1.5 \mathrm{~h})$ before $\mathrm{K}_{2} \mathrm{CO}_{3}$ was added. The reaction was stirred at $90^{\circ} \mathrm{C}$ for the time indicated in Table 2. Workup was performed as described in Method A.

3-Methyl-1-phenyl-5-nitro-1H-indazole (11a). Yield: $240 \mathrm{mg},(0.95 \mathrm{mmol}, 95 \%)$ as a brown solid, m.p. 115-117 ${ }^{\circ} \mathrm{C}$; IR: 1516, $1336 \mathrm{~cm}^{-1} ;{ }^{1} \mathrm{H}-\mathrm{NMR}\left(\mathrm{DMSO}_{-}\right)$): $\delta 8.89(\mathrm{~d}, J=2.2 \mathrm{~Hz}, 1 \mathrm{H}), 8.28(\mathrm{dd}, J=9.3$, 
$2.2 \mathrm{~Hz}, 1 \mathrm{H}), 7.93(\mathrm{~d}, J=9.3 \mathrm{~Hz}, 1 \mathrm{H}), 7.77(\mathrm{~d}, J=7.8 \mathrm{~Hz}, 2 \mathrm{H}), 7.62(\mathrm{t}, J=7.7 \mathrm{~Hz}, 2 \mathrm{H}), 7.46(\mathrm{t}, J=7.4 \mathrm{~Hz}, 1 \mathrm{H})$, $2.69(\mathrm{~s}, 3 \mathrm{H}) ;{ }^{13} \mathrm{C}-\mathrm{NMR}$ (DMSO- $\left.d_{6}\right): \delta 147.3,142.2,141.1,139.2,130.3,127.8,124.4,122.9,122.8,119.3$, 111.7, 12.1; MS: $m / z 253\left(\mathrm{M}^{+}\right)$, calculated $m / z$ 253.09. Anal. Calcd. for $\mathrm{C}_{14} \mathrm{H}_{11} \mathrm{~N}_{3} \mathrm{O}_{2}: \mathrm{C}, 66.40 ; \mathrm{H}, 4.38$; $\mathrm{N}, 16.59$. Found: $\mathrm{C}, 66.33 ; \mathrm{H}, 4.34 ; \mathrm{N}, 16.42$.

1-(2-Methoxyphenyl)-3-methyl-5-nitro-1H-indazole (11b). Yield: $232 \mathrm{mg}(0.82 \mathrm{mmol}, 82 \%)$ as a light brown solid, m.p. $128-130{ }^{\circ} \mathrm{C}$; IR: 2838, 1519, $1338 \mathrm{~cm}^{-1}$; ${ }^{1} \mathrm{H}-\mathrm{NMR}$ (DMSO- $d_{6}$ ): $\delta 8.86(\mathrm{~s}, 1 \mathrm{H})$, $8.21(\mathrm{~d}, J=9.3 \mathrm{~Hz}, 1 \mathrm{H}), 7.55(\mathrm{t}, J=8.0 \mathrm{~Hz}, 1 \mathrm{H}), 7.49(\mathrm{~d}, J=7.8 \mathrm{~Hz}, 1 \mathrm{H}), 7.38-7.28$ (overlapping signals, $2 \mathrm{H}), 7.16(\mathrm{t}, J=7.7 \mathrm{~Hz}, 1 \mathrm{H}), 3.78(\mathrm{~s}, 3 \mathrm{H}), 2.66(\mathrm{~s}, 3 \mathrm{H}) ;{ }^{13} \mathrm{C}-\mathrm{NMR}\left(\mathrm{DMSO}-d_{6}\right): \delta 154.0,146.6,143.0$, 141.9, 130.8, 128.6, 127.1, 123.2, 122.0, 121.4, 119.0, 113.4, 112.2, 56.2, 12.1; MS: $m / z 283\left(\mathrm{M}^{+}\right)$, calculated $m / z$ 283.10. Anal. Calcd. for $\mathrm{C}_{15} \mathrm{H}_{13} \mathrm{~N}_{3} \mathrm{O}_{3}$ : $\mathrm{C}, 63.60 ; \mathrm{H}, 4.63 ; \mathrm{N}, 14.83$. Found: $\mathrm{C}, 63.49 ; \mathrm{H}, 4.61 ; \mathrm{N}, 14.88$.

1-(3-Methoxyphenyl)-3-methyl-5-nitro-1H-indazole (11c). Yield: $229 \mathrm{mg}(0.81 \mathrm{mmol}, 81 \%)$ as a light brown solid, m.p. $105-106{ }^{\circ} \mathrm{C}$; IR: 2836, $1518,1338 \mathrm{~cm}^{-1}$; ${ }^{1} \mathrm{H}-\mathrm{NMR}$ (DMSO- $d_{6}$ ): $\delta 8.90(\mathrm{~s}, 1 \mathrm{H}$ ), $8.29(\mathrm{~d}, J=9.3 \mathrm{~Hz}, 1 \mathrm{H}), 7.96(\mathrm{~d}, J=9.3 \mathrm{~Hz}, 1 \mathrm{H}), 7.52(\mathrm{t}, J=8.1 \mathrm{~Hz}, 1 \mathrm{H}), 7.34(\mathrm{~d}, J=8.1 \mathrm{~Hz}, 1 \mathrm{H})$, $7.28(\mathrm{~s}, 1 \mathrm{H}), 7.04(\mathrm{~d}, J=8.3 \mathrm{~Hz}, 1 \mathrm{H}) ;{ }^{13} \mathrm{C}-\mathrm{NMR}$ (DMSO- $\left.d_{6}\right): \delta 160.6,147.3,142.3,141.2,140.3,131.2,124.4$, 122.9, 119.3, 114.9, 113.7, 111.9, 108.6, 56.0, 12.1; MS: $m / z 283\left(\mathrm{M}^{+}\right)$, calculated $m / z$ 283.10. Anal. Calcd. for $\mathrm{C}_{15} \mathrm{H}_{13} \mathrm{~N}_{3} \mathrm{O}_{3}$ : C, 63.60; H, 4.63; N, 14.83. Found: $\mathrm{C}, 63.52 ; \mathrm{H}, 4.55 ; \mathrm{N}, 14.71$.

1-(4-Methoxyphenyl)-3-methyl-5-nitro-1H-indazole (11d). Yield: $266 \mathrm{mg}(0.94 \mathrm{mmol}, 94 \%)$ as a tan solid, m.p. $159-160{ }^{\circ} \mathrm{C}$. IR: 2836, 1514, $1346 \mathrm{c} \mathrm{cm}^{-1} ;{ }^{1} \mathrm{H}-\mathrm{NMR}$ (DMSO- $\left.d_{6}\right): \delta 8.88(\mathrm{~d}, J=2.2 \mathrm{~Hz}, 1 \mathrm{H})$, $8.26(\mathrm{dd}, J=9.3,2.2 \mathrm{~Hz}, 1 \mathrm{H}), 7.79(\mathrm{~d}, J=9.3 \mathrm{~Hz}, 1 \mathrm{H}), 7.65(\mathrm{~d}, J=8.1 \mathrm{~Hz}, 2 \mathrm{H}), 7.15(\mathrm{~d}, J=8.1 \mathrm{~Hz}, 2 \mathrm{H})$, $3.85(\mathrm{~s}, 3 \mathrm{H}), 2.69(\mathrm{~s}, 3 \mathrm{H}) ;{ }^{13} \mathrm{C}-\mathrm{NMR}$ (DMSO- $\left.d_{6}\right): \delta 158.9,146.7,142.0,141.2,132.2,124.8,123.9,122.5$, 119.3, 1153, 111.4, 56.0, 12.1; MS: $m / z 283\left(\mathrm{M}^{+}\right)$, calculated $m / z$ 283.10. Anal. Calcd. for $\mathrm{C}_{15} \mathrm{H}_{13} \mathrm{~N}_{3} \mathrm{O}_{3}$ : C, 63.60; H, 4.63; N, 14.83. Found: C, 63.57; H, 4.60; N, 14.76 .

1-(4-Bromophenyl)-3-methyl-5-nitro-1H-indazole (11e). Yield: $314 \mathrm{mg},(0.95 \mathrm{mmol}, 95 \%)$ as a tan solid, m.p. 202-203 ${ }^{\circ} \mathrm{C}$; IR: 1512, $1345 \mathrm{~cm}^{-1}$; ${ }^{1} \mathrm{H}-\mathrm{NMR}$ (DMSO- $\left.d_{6}\right): \delta 8.90$ (d, $\left.J=2.2 \mathrm{~Hz}, 1 \mathrm{H}\right), 8.31$ (dd, $J=9.2$, $2.2 \mathrm{~Hz}, 1 \mathrm{H}), 7.96(\mathrm{~d}, J=9.2 \mathrm{~Hz}, 1 \mathrm{H}), 7.80(\mathrm{~d}, J=8.8 \mathrm{~Hz}, 2 \mathrm{H}), 7.75(\mathrm{~d}, J=8.8 \mathrm{~Hz}, 2 \mathrm{H}), 2.69(\mathrm{~s}, 3 \mathrm{H})$; ${ }^{13}$ C-NMR (DMSO- $d_{6}$ ): $\delta$ 147.7, 142.4, 141.1, 138.5, 133.1, 124.7, 124.6, 123.0, 120.1, 119.3, 111.8, 12.1; MS: $m / z 331\left(\mathrm{M}^{+}\right)$, calculated $m / z$ 331.00. Anal. Calcd. for $\mathrm{C}_{14} \mathrm{H}_{10} \mathrm{BrN}_{3} \mathrm{O}_{2}: \mathrm{C}, 50.62 ; \mathrm{H}, 3.03 ; \mathrm{N}, 12.65$. Found: $\mathrm{C}, 50.44 ; \mathrm{H}, 2.99 ; \mathrm{N}, 12.73$.

1-(3-Chlorophenyl)-3-methyl-5-nitro-1H-indazole (11f). Yield: $250 \mathrm{mg},(0.87 \mathrm{mmol}, 87 \%)$ as a tan solid, m.p. 135-136 ${ }^{\circ} \mathrm{C}$; IR: 1517, $1339 \mathrm{~cm}^{-1} ;{ }^{1} \mathrm{H}-\mathrm{NMR}$ (DMSO-d $): \delta 8.89$ (d, J = 2.2 Hz, $\left.1 \mathrm{H}\right), 8.31$ (dd, $J=9.3,2.2 \mathrm{~Hz}$, $1 \mathrm{H}), 7.98(\mathrm{~d}, J=9.3 \mathrm{~Hz}, 1 \mathrm{H}), 7.83(\mathrm{t}, J=2.2 \mathrm{~Hz}, 1 \mathrm{H}), 7.78(\mathrm{~d}, J=8.4 \mathrm{~Hz}, 1 \mathrm{H}), 7.64(\mathrm{t}, J=8.1 \mathrm{~Hz}, 1 \mathrm{H})$, $7.52(\mathrm{~d}, J=8.0 \mathrm{~Hz}, 1 \mathrm{H}), 2.69(\mathrm{~s}, 3 \mathrm{H}) ;{ }^{13} \mathrm{C}-\mathrm{NMR}\left(\mathrm{DMSO}-d_{6}\right): \delta 146.8,141.1,139.3,133.4,130.8,126.4$, 123.6, 122.0, 121.4, 120.1, 118.2, 110.9; MS: $m / z 287\left(\mathrm{M}^{+}\right)$, calculated $m / z$ 287.05. Anal. Calcd. for $\mathrm{C}_{14} \mathrm{H}_{10} \mathrm{ClN}_{3} \mathrm{O}_{2}: \mathrm{C}, 58.45 ; \mathrm{H}, 3.50 ; \mathrm{N}, 14.61$. Found: $\mathrm{C}, 58.58 ; \mathrm{H}, 3.59 ; \mathrm{N}, 14.53$.

1-(4-Chlorophenyl)-3-methyl-5-nitro-1H-indazole (11g). Yield: $267 \mathrm{mg}(0.93 \mathrm{mmol}, 93 \%)$ as a tan solid, m.p. 217-218 ${ }^{\circ} \mathrm{C}$; IR: 1511, $1339 \mathrm{~cm}^{-1},{ }^{1} \mathrm{H}-\mathrm{NMR}$ (DMSO- $d_{6}$ ): $\delta 8.91(\mathrm{~d}, J=2.2 \mathrm{~Hz}, 1 \mathrm{H}), 8.31$ (dd, $J=9.3,2.2 \mathrm{~Hz}$, $1 \mathrm{H}), 7.95(\mathrm{~d}, J=9.3 \mathrm{~Hz}, 1 \mathrm{H}), 7.82(\mathrm{~d}, J=8.7 \mathrm{~Hz}, 2 \mathrm{H}), 7.67(\mathrm{~d}, J=8.7 \mathrm{~Hz}, 2 \mathrm{H}), 2.69(\mathrm{~s}, 3 \mathrm{H}) ;{ }^{13} \mathrm{C}-\mathrm{NMR}$ (DMSO- $d_{6}$ ): $\delta 147.7,1424,141.1,138.1,131.8,130.2,124.6,124.5,123.0,119.4,111.8,12.1 ;$ MS: $\mathrm{m} / z$ $287\left(\mathrm{M}^{+}\right)$, calculated $m / z$ 287.05. Anal. Calcd. for $\mathrm{C}_{14} \mathrm{H}_{10} \mathrm{ClN}_{3} \mathrm{O}_{2}: \mathrm{C}, 58.45 ; \mathrm{H}, 3.50 ; \mathrm{N}, 14.61$. Found: $\mathrm{C}$, $58.42 ; \mathrm{H}, 3.49 ; \mathrm{N}, 14.48$.

1-(2,4-Dichlorophenyl)-3-methyl-5-nitro-1H-indazole (11h). Yield: $257 \mathrm{mg}(0.80 \mathrm{mmol}, 80 \%)$, m.p. $144-145^{\circ} \mathrm{C}$; IR: 1516, $1338 \mathrm{~cm}^{-1}$; ${ }^{1} \mathrm{H}-\mathrm{NMR}$ (DMSO- $\left.d_{6}\right): \delta 8.92(\mathrm{~d}, J=2.1 \mathrm{~Hz}, 1 \mathrm{H}), 8.27(\mathrm{dd}, J=9.2,2.1 \mathrm{~Hz}, 1 \mathrm{H})$, $8.01(\mathrm{~d}, J=2.2 \mathrm{~Hz}, 1 \mathrm{H}), 7.74(\mathrm{~d}, J=8.5 \mathrm{~Hz}, 1 \mathrm{H}), 7.69(\mathrm{dd}, J=8.5,2.2 \mathrm{~Hz}, 1 \mathrm{H}), 7.44(\mathrm{~d}, J=9.2 \mathrm{~Hz}, 1 \mathrm{H})$, 2.68 (s, 3H); ${ }^{13} \mathrm{C}-\mathrm{NMR}$ (DMSO- $d_{6}$ ): $\delta 147.5,143.1,142.4,135.3,135.0,132.0,131.5,130.7,129.3,123.5,122.8$, 119.3, 111.6, 12.1; MS: $m / z 321\left(\mathrm{M}^{+}\right)$, calculated $m / z$ 321.01. Anal. Calcd. for $\mathrm{C}_{14} \mathrm{H}_{9} \mathrm{Cl}_{2} \mathrm{~N}_{3} \mathrm{O}_{2}$ : C, 52.20; $\mathrm{H}, 2.82 ; \mathrm{N}, 13.04$. Found: $\mathrm{C}, 52.11 ; \mathrm{H}, 2.91 ; \mathrm{N}, 13.09$. 
3-Methyl-5-nitro-1-(3-(trifluoromethyl)phenyl)-1H-indazole (11i). Yield: $282 \mathrm{mg}(0.88 \mathrm{mmol}, 88 \%)$ as a white solid, m.p. $112-113{ }^{\circ} \mathrm{C}$; IR: 1520, $1339 \mathrm{~cm}^{-1} ;{ }^{1} \mathrm{H}-\mathrm{NMR}$ (DMSO- $d_{6}$ ): $\delta 8.90(\mathrm{~s}, 1 \mathrm{H})$, $8.32(\mathrm{~d}, J=9.3 \mathrm{~Hz}, 1 \mathrm{H}), 8.13(\mathrm{~d}, J=7.8 \mathrm{~Hz}, 1 \mathrm{H}), 8.07(\mathrm{~s}, 1 \mathrm{H}), 7.99(\mathrm{~d}, J=9.3 \mathrm{~Hz}, 1 \mathrm{H}), 7.90-7.77$ (complex, 2H), $2.70(\mathrm{~s}, 3 \mathrm{H}) ;{ }^{13} \mathrm{C}-\mathrm{NMR}$ (DMSO- $\left.d_{6}\right): \delta 148.0,142.5,141.1,139.8,131.6,130.9$ (q, $\left.J=32.6 \mathrm{~Hz}\right), 126.2$, $124.8,124.2(\mathrm{q}, J=272.6 \mathrm{~Hz}), 124.0(\mathrm{q}, J=6.7 \mathrm{~Hz}), 123.1,119.2(2 \mathrm{C}), 111.8,12.0 ; \mathrm{MS}: m / z 321\left(\mathrm{M}^{+}\right)$, calculated $m / z$ 321.07. Anal. Calcd. for $\mathrm{C}_{15} \mathrm{H}_{10} \mathrm{~F}_{3} \mathrm{~N}_{3} \mathrm{O}_{2}: \mathrm{C}, 56.08 ; \mathrm{H}, 3.14 ; \mathrm{N}, 13.08$. Found: $\mathrm{C}, 56.02$; $\mathrm{H}, 3.18 ; \mathrm{N}, 13.11$.

3-Methyl-5-nitro-1-(4-(trifluoromethyl)phenyl)-1H-indazole (11j). Yield: $225 \mathrm{mg},(0.70 \mathrm{mmol}, 70 \%)$ as a white solid, mp $167-168{ }^{\circ} \mathrm{C}$; IR: 1525, $1331 \mathrm{~cm}^{-1} ;{ }^{1} \mathrm{H}-\mathrm{NMR}$ (DMSO- $\left.d_{6}\right): \delta 8.89(\mathrm{~d}, J=2.2 \mathrm{~Hz}, 1 \mathrm{H}$ ), $8.31(\mathrm{dd}, J=9.3,2.2 \mathrm{~Hz}, 1 \mathrm{H}), 8.05(\mathrm{~d}, J=9.3 \mathrm{~Hz}, 1 \mathrm{H}), 8.02(\mathrm{~d}, J=8.6 \mathrm{~Hz}, 2 \mathrm{H}), 7.95(\mathrm{~d}, J=8.6 \mathrm{~Hz}$, 2H), $2.69(\mathrm{~s}, 3 \mathrm{H}) ;{ }^{13} \mathrm{C}-\mathrm{NMR}$ (DMSO- $\left.d_{6}\right): \delta 148.3,142.6,142.3,141.1,127.4(\mathrm{q}, J=4.0 \mathrm{~Hz}), 127.1,125.1$, $124.5(\mathrm{q}, J=269.1 \mathrm{~Hz}), 123.2,122.6,119.2,112.0,12.1 ; \mathrm{MS}: m / z 321\left(\mathrm{M}^{+}\right)$. calculated $m / z$ 321.07. Anal. Calcd. for $\mathrm{C}_{15} \mathrm{H}_{10} \mathrm{~F}_{3} \mathrm{~N}_{3} \mathrm{O}_{2}$ : C, 56.08; H, 3.14; N, 13.08. Found: $\mathrm{C}, 55.98 ; \mathrm{H}, 3.19 ; \mathrm{N}, 13.04$.

1-(4-Cyanophenyl)-3-methyl-5-nitro-1H-indazole (11k). Yield: $222 \mathrm{mg}(0.80 \mathrm{mmol}, 80 \%)$ as a brown solid, m.p. $\geq 194{ }^{\circ} \mathrm{C}$ (sub), $\geq 260{ }^{\circ} \mathrm{C}$ (dec); IR: 2226, 1516, $1338 \mathrm{~cm}^{-1} ;{ }^{1} \mathrm{H}-\mathrm{NMR}$ (DMSO- $d_{6}$ ): $\delta 8.93(\mathrm{~d}, J=2.2 \mathrm{~Hz}, 1 \mathrm{H}), 8.35(\mathrm{dd}, J=9.1,2.2 \mathrm{~Hz}, 1 \mathrm{H}), 8.11(\mathrm{~d}, J=9.1 \mathrm{~Hz}, 1 \mathrm{H}), 8.08(\mathrm{~d}, J=8.9 \mathrm{~Hz}, 2 \mathrm{H})$, $8.04(\mathrm{~d}, J=8.9 \mathrm{~Hz}, 2 \mathrm{H}), 2.71(\mathrm{~s}, 3 \mathrm{H}) ;{ }^{13} \mathrm{C}-\mathrm{NMR}\left(\mathrm{DMSO}-d_{6}\right): \delta 148.7,142.8,142.7,141.1,134.5,125.3$, 123.3, 122.6, 119.3, 118.9, 112.3, 109.4, 12.1; MS: $m / z 278\left(\mathrm{M}^{+}\right)$, calculated $m / z$ 278.08. Anal. Calcd. for $\mathrm{C}_{15} \mathrm{H}_{10} \mathrm{~N}_{4} \mathrm{O}_{2}$ : C, 64.74; $\mathrm{H}, 3.62 ; \mathrm{N}, 20.13$. Found: $\mathrm{C}, 64.69 ; \mathrm{H}, 3.67 ; \mathrm{N}, 20.06$.

4-(3-Methyl-5-nitro-1H-indazol-1-yl)benzenesulfonamide (111). Yield: $249 \mathrm{mg}(0.75 \mathrm{mmol}, 75 \%)$, m.p. 265-266 ${ }^{\circ} \mathrm{C}$; IR: 3422, 1515, 1331, 1320, $1123 \mathrm{~cm}^{-1} ;{ }^{1} \mathrm{H}-\mathrm{NMR}$ (DMSO- $\left.d_{6}\right): \delta 8.92(\mathrm{~d}, J=2.2 \mathrm{~Hz}, 1 \mathrm{H}$ ), $8.34(\mathrm{dd}, J=9.3,2.2 \mathrm{~Hz}, 1 \mathrm{H}), 8.09(\mathrm{~d}, J=9.3 \mathrm{~Hz}, 1 \mathrm{H}), 8.04(\mathrm{~d}, J=8.4 \mathrm{~Hz}, 2 \mathrm{H}), 8.01(\mathrm{~d}, J=8.4 \mathrm{~Hz}, 2 \mathrm{H})$, $7.51(\mathrm{~s}, 2 \mathrm{H}), 2.71(\mathrm{~s}, 3 \mathrm{H}) ;{ }^{13} \mathrm{C}-\mathrm{NMR}\left(\mathrm{DMSO}-d_{6}\right): \delta 148.2,142.54,145.50,141.6,141.0,127.9,125.0,123.1$, 122.3, 119.2, 112.0, 12.0; MS: $m / z 332\left(\mathrm{M}^{+}\right)$, calculated $m / z$ 332.06. Anal. Calcd. for $\mathrm{C}_{14} \mathrm{H}_{12} \mathrm{~N}_{4} \mathrm{O}_{4} \mathrm{~S}$ : C, 50.60; H, 3.64; N, 16.86. Found: C, 50.70; H, 3.63; N, 16.78.

4-(3-Methyl-5-nitro-1H-indazol-1-yl)benzoic acid (11m). Yield: $208 \mathrm{mg}(0.70 \mathrm{mmol}, 70 \%)$ as a tan solid, m.p. $\geq 240{ }^{\circ} \mathrm{C}$ (sub), $\geq 320{ }^{\circ} \mathrm{C}$ (dec). IR: 3441-2352, 1697, 1514, $1346 \mathrm{~cm}^{-1} ;{ }^{1} \mathrm{H}-\mathrm{NMR}$ (DMSO- $d_{6}$ ): $\delta 12.8(\mathrm{br} \mathrm{s}, 1 \mathrm{H}), 8.93(\mathrm{~d}, J=2.2 \mathrm{~Hz}, 1 \mathrm{H}), 8.34(\mathrm{dd}, J=9.3,2.2 \mathrm{~Hz}, 1 \mathrm{H}), 8.16(\mathrm{~d}, J=8.6 \mathrm{~Hz}, 2 \mathrm{H})$, $8.09(\mathrm{~d}, J=9.3, \mathrm{~Hz}, 1 \mathrm{H}), 7.95(\mathrm{~d}, J=8.6 \mathrm{~Hz}, 2 \mathrm{H}), 2.71(\mathrm{~s}, 3 \mathrm{H}) ;{ }^{13} \mathrm{C}-\mathrm{NMR}$ (DMSO- $\left.d_{6}\right): \delta 167.1,148.2,142.6$, 142.5, 141.1, 131.5, 125.0, 123.1, 122.0, 119.4, 119.3, 112.2, 12.1; MS: $m / z 297\left(\mathrm{M}^{+}\right)$, calculated $m / z$ 297.07. Anal. Calcd. for $\mathrm{C}_{15} \mathrm{H}_{11} \mathrm{~N}_{3} \mathrm{O}_{4}$ : $\mathrm{C}, 60.61 ; \mathrm{H}, 3.73 ; \mathrm{N}, 14.14$. Found: $\mathrm{C}, 60.53 ; \mathrm{H}, 3.68 ; \mathrm{N}, 14.22$.

1-Phenyl-5-nitro-1H-indazole (12a). Yield: $172 \mathrm{mg}(0.72 \mathrm{mmol}, 72 \%)$ as a tan solid, m.p. $178-180{ }^{\circ} \mathrm{C}$ (lit m.p. 178-180 ${ }^{\circ} \mathrm{C}$ [18]); IR: 1534, $1341 \mathrm{~cm}^{-1} ;{ }^{1} \mathrm{H}-\mathrm{NMR}$ (DMSO-d $\mathrm{d}_{6}$ ): $\delta 8.95$ (s, 1H), $8.70(\mathrm{~s}, 1 \mathrm{H}$ ), $8.30(\mathrm{~d}, J=9.3 \mathrm{~Hz}, 1 \mathrm{H}), 7.98(\mathrm{~d}, J=9.3 \mathrm{~Hz}, 1 \mathrm{H}), 7.81(\mathrm{~d}, J=7.9 \mathrm{~Hz}, 2 \mathrm{H}), 7.65(\mathrm{~d}, J=7.7 \mathrm{~Hz}, 2 \mathrm{H})$, $7.51(\mathrm{t}, J=7.4 \mathrm{~Hz}, 1 \mathrm{H}) ;{ }^{13} \mathrm{C}-\mathrm{NMR}\left(\mathrm{DMSO}-d_{6}\right): \delta 142.8,140.5,139.1,138.9,130.3,128.2,124.8,123.3,122.7$, 119.9, 111.9; MS: $m / z 239\left(\mathrm{M}^{+}\right)$, calculated $m / z$ 239.07. Anal. Calcd. for $\mathrm{C}_{13} \mathrm{H}_{9} \mathrm{~N}_{3} \mathrm{O}_{2}: \mathrm{C}, 65.27 ; \mathrm{H}, 3.79$; N, 17.56. Found: C, 65.16; H, 3.77; N, 17.57 .

1-(2-Methoxyphenyl)-5-nitro-1H-indazole (12b). This reaction stopped at the hydrazone stage and did not give an indazole.

1-(3-Methoxyphenyl)-5-nitro-1H-indazole (12c): Yield: $180 \mathrm{mg}(0.67 \mathrm{mmol}, 67 \%)$ as a yellow solid, m.p. 132-133 ${ }^{\circ} \mathrm{C}$ (lit [36] m.p. $135^{\circ} \mathrm{C}$ ); IR: 1516, $1344 \mathrm{~cm}^{-1} ;{ }^{1} \mathrm{H}-\mathrm{NMR}$ (DMSO-d $\left.d_{6}\right): \delta 8.94(\mathrm{~s}, 1 \mathrm{H}), 8.69(\mathrm{~s}, 1 \mathrm{H})$, $8.30(\mathrm{~d}, J=9.2 \mathrm{~Hz}, 1 \mathrm{H}), 8.01(\mathrm{~d}, J=9.3 \mathrm{~Hz}, 1 \mathrm{H}), 7.55(\mathrm{t}, J=8.0 \mathrm{~Hz}, 1 \mathrm{H}), 7.37(\mathrm{~d}, J=8.0 \mathrm{~Hz}, 1 \mathrm{H})$, $7.32(\mathrm{~s}, 1 \mathrm{H}), 7.08(\mathrm{~d}, J=8.2 \mathrm{~Hz}, 1 \mathrm{H}), 3.87(\mathrm{~s}, 3 \mathrm{H}) ;{ }^{13} \mathrm{C}-\mathrm{NMR}\left(\mathrm{DMSO}-d_{6}\right): \delta 160.6,142.8,140.5,140.2$, $130.8,131.2,124.8,122.7,119.8,115.3,114.1,112.0,109.0,56.0 ;$ MS: $m / z 269\left(\mathrm{M}^{+}\right)$, calculated $m / z 269.08$. Anal. Calcd. for $\mathrm{C}_{14} \mathrm{H}_{11} \mathrm{~N}_{3} \mathrm{O}_{3}: \mathrm{C}, 62.45 ; \mathrm{H}, 4.12 ; \mathrm{N}, 15.61$. Found: $\mathrm{C}, 62.37 ; \mathrm{H}, 4.06 ; \mathrm{N}, 15.74$. 
1-(4-Methoxyphenyl)-5-nitro-1H-indazole (12d). Yield: $199 \mathrm{mg}(0.74 \mathrm{mmol}, 74 \%)$ as a light yellow solid, m.p. $181-182{ }^{\circ} \mathrm{C}$; IR: 1516, $1342 \mathrm{~cm}^{-1} ;{ }^{1} \mathrm{H}-\mathrm{NMR}$ (DMSO-d $\left.\mathrm{d}_{6}\right): \delta 8.92(\mathrm{~d}, J=2.2 \mathrm{~Hz}, 1 \mathrm{H}), 8.64(\mathrm{~s}, 1 \mathrm{H})$, $8.27(\mathrm{~d}, J=9.2,2.2 \mathrm{~Hz}, 1 \mathrm{H}), 7.84(\mathrm{~d}, J=9.2 \mathrm{~Hz}, 1 \mathrm{H}), 7.68(\mathrm{~d}, J=8.7 \mathrm{~Hz}, 2 \mathrm{H}), 7.18(\mathrm{~d}, J=8.7 \mathrm{~Hz}, 2 \mathrm{H})$, $3.86(\mathrm{~s}, 3 \mathrm{H}) ;{ }^{13} \mathrm{C}-\mathrm{NMR}$ (DMSO- $\left.d_{6}\right): \delta 159.2,142.6,141.6,138.3,132.1,125.2,124.3,122.4,119.8,115.6$, 111.6, 56.2; MS: $m / z 269\left(\mathrm{M}^{+}\right)$, calculated $m / z$ 269.08. Anal. Calcd. for $\mathrm{C}_{14} \mathrm{H}_{11} \mathrm{~N}_{3} \mathrm{O}_{3}: \mathrm{C}, 62.45 ; \mathrm{H}, 4.12$; $\mathrm{N}, 15.61$. Found: $\mathrm{C}, 62.41 ; \mathrm{H}, 4.07 ; \mathrm{N}, 15.56$.

1-(4-Bromophenyl)-5-nitro-1H-indazole (12e). Yield: $222 \mathrm{mg}(0.70 \mathrm{mmol}, 70 \%)$ as a tan solid, m.p. 169-170 ${ }^{\circ} \mathrm{C}$; IR: 1511, $1348 \mathrm{~cm}^{-1} ;{ }^{1} \mathrm{H}-\mathrm{NMR}$ (DMSO- $d_{6}$ ): $\delta 8.95$ (s, 1H), $8.71(\mathrm{~s}, 1 \mathrm{H}), 8.32(\mathrm{~d}, J=9.3 \mathrm{~Hz}$, $1 \mathrm{H}), 8.01(\mathrm{~d}, J=9.2 \mathrm{~Hz}, 1 \mathrm{H}), 7.84(\mathrm{~d}, J=8.4 \mathrm{~Hz}, 2 \mathrm{H}), 7.79(\mathrm{~d}, J=8.4 \mathrm{~Hz}, 2 \mathrm{H}) ;{ }^{13} \mathrm{C}-\mathrm{NMR}\left(\mathrm{DMSO}-d_{6}\right)$ : $\delta 142.9,140.4,139.2,138.4,133.2,125.2,125.0,122.8,120.7,119.9,111.9 ;$ MS: $m / z 317\left(\mathrm{M}^{+}\right)$, calculated $m / z$ 316.98. Anal. Calcd. for $\mathrm{C}_{13} \mathrm{H}_{8} \mathrm{BrN}_{3} \mathrm{O}_{2}: \mathrm{C}, 49.08 ; \mathrm{H}, 2.53 ; \mathrm{N}, 13.21$. Found: $\mathrm{C}, 48.97 ; \mathrm{H}, 2.59$; $\mathrm{N}, 13.25$.

1-(3-Chlorophenyl)-5-nitro-1H-indazole (12f). Yield: $164 \mathrm{mg}(0.60 \mathrm{mmol}, 60 \%)$ as an off-white solid, m.p. 143-144 ${ }^{\circ} \mathrm{C}$; IR: 1514, $1346 \mathrm{~cm}^{-1} ;{ }^{1} \mathrm{H}-\mathrm{NMR}$ (DMSO- $\left.d_{6}\right): \delta 8.95(\mathrm{~s}, 1 \mathrm{H}), 8.72(\mathrm{~s}, 1 \mathrm{H}), 8.32(\mathrm{~d}, J=9.2 \mathrm{~Hz}, 1 \mathrm{H})$, $8.03(\mathrm{~d}, J=9.2 \mathrm{~Hz}, 1 \mathrm{H}), 7.88(\mathrm{~s}, 1 \mathrm{H}), 7.82(\mathrm{~d}, J=8.0 \mathrm{~Hz}, 1 \mathrm{H}), 7.67(\mathrm{t}, J=8.1 \mathrm{~Hz}, 1 \mathrm{H}), 7.57(\mathrm{~d}, J=8.2 \mathrm{~Hz}$, $1 \mathrm{H}) ;{ }^{13} \mathrm{C}-\mathrm{NMR}$ (DMSO- $d_{6}$ ): $\delta 142.9,140.5,140.3,139.4 .134 .6,132.0,128.0,125.0,123.0,122.9,121.7$, 119.8, 112.0; MS: $m / z 273\left(\mathrm{M}^{+}\right)$, calculated $m / z$ 273.03. Anal. Calcd. for $\mathrm{C}_{13} \mathrm{H}_{8} \mathrm{ClN}_{3} \mathrm{O}_{2}: \mathrm{C}, 57.05 ; \mathrm{H}, 2.95$; N, 15.35. Found: C, 56.96; H, 3.00; N, 15.29 .

1-(4-Chlorophenyl)-5-nitro-1H-indazole (12g). Yield: $191 \mathrm{mg}(0.70 \mathrm{mmol}, 70 \%)$ as a light yellow solid, m.p. $179-180{ }^{\circ} \mathrm{C}$; IR: $1509,1343 \mathrm{~cm}^{-1} ;{ }^{1} \mathrm{H}-\mathrm{NMR}$ (DMSO- $\left.d_{6}\right): \delta 8.95(\mathrm{~d}, J=2.2 \mathrm{~Hz}, 1 \mathrm{H}), 8.71(\mathrm{~s}, 1 \mathrm{H})$, $8.31(\mathrm{dd}, J=9.3,2.2 \mathrm{~Hz}, 1 \mathrm{H}), 7.99(\mathrm{~d}, J=9.3 \mathrm{~Hz}, 1 \mathrm{H}), 7.85(\mathrm{~d}, J=8.6 \mathrm{~Hz}, 2 \mathrm{H}), 7.70(\mathrm{~d}, J=8.6 \mathrm{~Hz}, 2 \mathrm{H})$; ${ }^{13}$ C-NMR (DMSO- $d_{6}$ ): $\delta 142.9,140.5,139.2,138.0,132.4,130.3,124.9,122.8,119.9,111.9$ (one C not observed); MS: $m / z 273\left(\mathrm{M}^{+}\right)$, calculated $m / z$ 273.03. Anal. Calcd. for $\mathrm{C}_{13} \mathrm{H}_{8} \mathrm{ClN}_{3} \mathrm{O}_{2}: \mathrm{C}, 57.05 ; \mathrm{H}, 2.95$; $\mathrm{N}, 15.35$. Found: $\mathrm{C}, 57.12 ; \mathrm{H}, 2.97 ; \mathrm{N}, 15.33$.

1-(2,4-Dichlorophenyl)-5-nitro-1H-indazole (12h). Yield: $215 \mathrm{mg}(0.70 \mathrm{mmol}, 70 \%)$ as a light pink solid, m.p. $101-102{ }^{\circ} \mathrm{C}$. IR: 1517, $1344 \mathrm{~cm}^{-1} ;{ }^{1} \mathrm{H}-\mathrm{NMR}$ (DMSO-d $\left.)_{6}\right): \delta 8.96(\mathrm{~s}, 1 \mathrm{H}), 8.72(\mathrm{~s}, 1 \mathrm{H})$, $8.29(\mathrm{~d}, J=9.2 \mathrm{~Hz}, 1 \mathrm{H}), 8.03(\mathrm{~s}, 1 \mathrm{H}), 7.78(\mathrm{~d}, J=8.3 \mathrm{~Hz}, 1 \mathrm{H}), 7.73(\mathrm{~d}, J=8.3 \mathrm{~Hz}, 1 \mathrm{H})$, $7.52(\mathrm{~d}, J=9.2 \mathrm{~Hz}, 1 \mathrm{H}) ;{ }^{13} \mathrm{C}-\mathrm{NMR}$ (DMSO- $\left.d_{6}\right): \delta 143.0,142.3,139.2,135.7,135.0,132.2,131.6$, 130.8, 129.3, 123.9, 122.7, 119.8, 111.7; MS: $m / z 307\left(\mathrm{M}^{+}\right)$, calculated $m / z$ 306.99. Anal. Calcd. for $\mathrm{C}_{13} \mathrm{H}_{7} \mathrm{Cl}_{2} \mathrm{~N}_{3} \mathrm{O}_{2}$ : C, 50.68; H, 2.29; N, 13.64. Found: C, 50.67; H, 2.34; N, 13.53 .

1-(3-(Trifluoromethyl)phenyl)-5-nitro-1H-indazole (12i). Yield: $190 \mathrm{mg}(0.62 \mathrm{mmol}, 62 \%)$ as a light yellow solid, m.p. $118-119{ }^{\circ} \mathrm{C}$. IR: 1513, $1347 \mathrm{~cm}^{-1}$; ${ }^{1} \mathrm{H}-\mathrm{NMR}$ (DMSO- $d_{6}$ ): $\delta 8.97(\mathrm{~s}, 1 \mathrm{H})$, $8.75(\mathrm{~s}, 1 \mathrm{H}), 8.35(\mathrm{~d}, J=9.2 \mathrm{~Hz}, 1 \mathrm{H}), 8.18(\mathrm{~d}, J=7.2 \mathrm{~Hz}, 1 \mathrm{H}), 8.13(\mathrm{~s}, 1 \mathrm{H}), 8.05(\mathrm{~d}, J=9.3 \mathrm{~Hz}, 1 \mathrm{H})$, 7.94-7.85 (complex, $2 \mathrm{H}) ;{ }^{13} \mathrm{C}-\mathrm{NMR}\left(\mathrm{DMSO}-d_{6}\right): \delta 143.0,140.6,139.8,139.6,131.7,131.0(\mathrm{q}, J=32.7 \mathrm{~Hz})$, $126.9,125.2,124.6(\mathrm{q}, J=4.3 \mathrm{~Hz}), 124.1(\mathrm{q}, J=272.6 \mathrm{~Hz}), 123.0,119.8(\mathrm{q}, J=4.9 \mathrm{~Hz}$ ), 112.0 (one $\mathrm{C}$ not observed); MS: $m / z 307\left(\mathrm{M}^{+}\right)$, calculated $m / z$ 307.06. Anal. Calcd. for $\mathrm{C}_{14} \mathrm{H}_{8} \mathrm{~F}_{3} \mathrm{~N}_{3} \mathrm{O}_{2}: \mathrm{C}, 54.73 ; \mathrm{H}, 2.62$; $\mathrm{N}, 13.68$. Found: $\mathrm{C}, 54.66 ; \mathrm{H}, 2.64 ; \mathrm{N}, 13.57$.

1-(4-(Trifluoromethyl)phenyl)-5-nitro-1H-indazole (12j). Yield: $209 \mathrm{mg}(0.68 \mathrm{mmol}, 68 \%)$ as a light yellow solid, m.p. $151-152{ }^{\circ} \mathrm{C}$. IR: 1519, $1327 \mathrm{~cm}^{-1}$; ${ }^{1} \mathrm{H}-\mathrm{NMR}$ (DMSO- $\left.d_{6}\right): \delta 8.95$ (s, 1H), $8.76(\mathrm{~s}, 1 \mathrm{H}$ ), $8.34(\mathrm{~d}, J=9.5 \mathrm{~Hz}, 1 \mathrm{H}), 8.11$ (obscured d, $J=9.5 \mathrm{~Hz}, 1 \mathrm{H}), 8.08(\mathrm{~d}, J=8.5 \mathrm{~Hz}, 2 \mathrm{H}), 8.00(\mathrm{~d}, J=8.5 \mathrm{~Hz}, 2 \mathrm{H})$; ${ }^{13}$ C-NMR (DMSO- $\left.d_{6}\right): \delta 143.1,142.3,140.5,139.8,127.9,(q, J=32.3 \mathrm{~Hz}), 127.5(\mathrm{q}, J=3.5 \mathrm{~Hz}), 125.4$, $124.4(\mathrm{q}, J=273.7 \mathrm{~Hz}), 123.3,123.1,119.9,112.1 ; \mathrm{MS}: \mathrm{m} / z 307\left(\mathrm{M}^{+}\right)$, calculated $m / z$ 307.06. Anal. Calcd. for $\mathrm{C}_{14} \mathrm{H}_{8} \mathrm{~F}_{3} \mathrm{~N}_{3} \mathrm{O}_{2}: \mathrm{C}, 54.73 ; \mathrm{H}, 2.62 ; \mathrm{N}, 13.68$. Found: $\mathrm{C}, 54.68 ; \mathrm{H}, 2.61 ; \mathrm{N}, 13.64$.

1-(4-Cyanophenyl)-5-nitro-1H-indazole (12k). Yield: $158 \mathrm{mg}(0.60 \mathrm{mmol}, 60 \%)$ as a light yellow solid, m.p. 250-251 ${ }^{\circ} \mathrm{C}$. IR: 2227, 1512, $1344 \mathrm{~cm}^{-1} ;{ }^{1} \mathrm{H}-\mathrm{NMR}$ (DMSO- $\left.d_{6}\right): \delta 8.97(\mathrm{~d}, J=2.2 \mathrm{~Hz}, 1 \mathrm{H}), 8.78(\mathrm{~s}, 1 \mathrm{H})$, $8.36(\mathrm{dd}, J=9.3,2.2 \mathrm{~Hz}, 1 \mathrm{H}), 8.15(\mathrm{~d}, J=9.3 \mathrm{~Hz}, 1 \mathrm{H}), 8.12(\mathrm{~d}, J=8.8 \mathrm{~Hz}, 2 \mathrm{H}), 8.07(\mathrm{~d}, J=8.8 \mathrm{~Hz}, 2 \mathrm{H})$; 
${ }^{13}$ C-NMR (DMSO-d $): \delta 142.1,141.6,139.4,139.1,133.6,124.5,122.1,120.0,118.9,117.7,111.3,109.0$; MS: $m / z 264\left(\mathrm{M}^{+}\right)$, calculated $m / z$ 264.06. Anal. Calcd. for $\mathrm{C}_{14} \mathrm{H}_{8} \mathrm{~N}_{4} \mathrm{O}_{2}$ : C, 63.64; $\mathrm{H}, 3.05 ; \mathrm{N}, 21.20$. Found: C, 63.55; H, 3.09; N, 21.08.

4-(5-Nitro-1H-indazol-1-yl)benzenesulfonamide (121). Yield: $159 \mathrm{mg}(0.50 \mathrm{mmol}, 50 \%), \mathrm{m} . \mathrm{p} .237-238{ }^{\circ} \mathrm{C}$; IR: 3329, 3241, 1512, $1339 \mathrm{~cm}^{-1} ;{ }^{1} \mathrm{H}-\mathrm{NMR}\left(\mathrm{DMSO}-d_{6}\right): \delta 8.97(\mathrm{~d}, J=2.2 \mathrm{~Hz}, 1 \mathrm{H}), 8.76(\mathrm{~s}, 1 \mathrm{H})$, $8.35(\mathrm{dd}, J=9.3,2.2 \mathrm{~Hz}, 1 \mathrm{H}), 8.13(\mathrm{~d}, J=9.3 \mathrm{~Hz}, 1 \mathrm{H}), 8.06(\mathrm{~s}, 4 \mathrm{H}), 7.53(\mathrm{br} \mathrm{s}, 2 \mathrm{H}) ;{ }^{13} \mathrm{C}-\mathrm{NMR}\left(\mathrm{DMSO}_{6}\right)$ : $\delta$ 143.1, 141.60, 141.55, 140.5, 139.8, 128.0, 125.3,123.1, 122.2, 119.9, 112.2; MS: $m / z 318\left(\mathrm{M}^{+}\right)$, calculated $m / z$ 318.04. Anal. Calcd. for $\mathrm{C}_{13} \mathrm{H}_{10} \mathrm{~N}_{4} \mathrm{O}_{4} \mathrm{~S}$ : C, 49.05; $\mathrm{H}, 3.17 ; \mathrm{N}, 17.60$. Found: C, 49.11; $\mathrm{H}, 3.16$; $\mathrm{N}, 17.66$.

4-(5-Nitro-1H-indol-1yl)benzoic acid $\mathbf{( 1 2} \mathbf{m})$. This product was formed only using the two-step procedure. Yield: $142 \mathrm{mg}(0.50 \mathrm{mmol}, 50 \%)$ as a brown product, m.p. 158-159 ${ }^{\circ} \mathrm{C}$; IR: 3395-2372, 1694, 1518, $1345 \mathrm{~cm}^{-1}$; ${ }^{1} \mathrm{H}-\mathrm{NMR}$ (DMSO-d 6 ): $\delta 13.3$ (br s, $\left.1 \mathrm{H}\right), 8.97$ (d, J = $\left.2.2 \mathrm{~Hz}, 1 \mathrm{H}\right), 8.76(\mathrm{~s}, 1 \mathrm{H})$, $8.35(\mathrm{dd}, J=9.3,2.2 \mathrm{~Hz}, 1 \mathrm{H}), 8.18(\mathrm{~d}, J=8.4 \mathrm{~Hz}, 2 \mathrm{H}), 8.13(\mathrm{~d}, J=9.3 \mathrm{~Hz}, 1 \mathrm{H}), 7.98(\mathrm{~d}, J=8.4 \mathrm{~Hz}, 2 \mathrm{H})$; ${ }^{13}$ C-NMR (DMSO- $d_{6}$ ): $\delta$ 167.0, 143.0, 142.5, 140.4, 139.7, 131.5, 129.8, 125.3, 123.0, 122.5, 119.9, 112.2; MS: $m / z 283\left(\mathrm{M}^{+}\right)$, calculated $m / z$ 283.06. Anal. Calcd. for $\mathrm{C}_{14} \mathrm{H}_{9} \mathrm{~N}_{3} \mathrm{O}_{4}$ : C, 59.37; $\mathrm{H}, 3.20 ; \mathrm{N}, 14.84$. Found: C, 59.32; H, 3.23; N, 14.75 .

\subsection{Representative Procedure for the General Indazole Synthesis}

To a stirred solution of the carbonyl compound $(\mathbf{1 3}, \mathbf{1 4}$ or $\mathbf{1 5}, 1.0 \mathrm{mmol})$ in DMPU (5 mL) were added powdered $4 \AA$ molecular sieves ( $30 \mathrm{wt} \%$ relative to the carbonyl substrate) and $\mathrm{ArNHNH} 2 \cdot \mathrm{HCl}$ ( $1.5 \mathrm{mmol})$. The mixture was heated at $90{ }^{\circ} \mathrm{C}$ (oil bath) for $1.5 \mathrm{~h}$ at which time $\mathrm{CuI}(0.2 \mathrm{mmol})$ and $\mathrm{K}_{2} \mathrm{CO}_{3}(2.5 \mathrm{mmol})$ were added and heating was continued at this temperature for $16 \mathrm{~h}$. The crude reaction mixture was cooled to $23^{\circ} \mathrm{C}$ and filtered through a Celite ${ }^{\circledR}$ pad (Fisher Scientific, Pittsburgh, PA, USA). The pad was rinsed with ether $(2 \times 20 \mathrm{~mL})$ and the combined filtrate was washed with water $(25 \mathrm{~mL})$, saturated $\mathrm{NaCl}(25 \mathrm{~mL})$, dried $\left(\mathrm{MgSO}_{4}\right)$, filtered, and concentrated under vacuum. The products were purified by silica gel chromatography using increasing concentrations of EtOAc in hexanes. Yields as well as physical and spectral data are given below.

3-Methyl-1-phenyl-1H-indazole (16a). Yield: $169 \mathrm{mg}(0.81 \mathrm{mmol}, 81 \%)$ as tan solid, m.p. $73-74{ }^{\circ} \mathrm{C}$ (lit [18] m.p. $\left.73-74{ }^{\circ} \mathrm{C}\right)$; IR: 1597, $1505 \mathrm{~cm}^{-1}$; ${ }^{1} \mathrm{H}-\mathrm{NMR}\left(\mathrm{CDCl}_{3}\right)$ : $\delta 7.75-7.96$ (complex, 4H), $7.52(\mathrm{t}, J=7.3 \mathrm{~Hz}, 2 \mathrm{H}), 7.42(\mathrm{t}, J=7.5 \mathrm{~Hz}, 1 \mathrm{H}), 7.32(\mathrm{t}, J=7.3 \mathrm{~Hz}, 1 \mathrm{H}), 7.21(\mathrm{t}, J=7.5 \mathrm{~Hz}, 1 \mathrm{H}), 2.65(\mathrm{~s}, 3 \mathrm{H})$; ${ }^{13} \mathrm{C}-\mathrm{NMR}\left(\mathrm{CDCl}_{3}\right): \delta 144.0,140.3,139.5,129.4,127.1,126.1,124.9,122.4,120.8,120.6,110.3,12.0 ; \mathrm{MS}: \mathrm{m} / \mathrm{z}$ $208\left(\mathrm{M}^{+}\right)$, calculated $m / z$ 208.10. Anal. Calcd. for $\mathrm{C}_{14} \mathrm{H}_{12} \mathrm{~N}_{2}: \mathrm{C}, 80.74 ; \mathrm{H}, 5.81 ; \mathrm{N}, 13.45$. Found: C, 81.01; $\mathrm{H}, 6.08 ; \mathrm{N}, 13.23$.

1-(4-Methoxyphenyl)-3-methyl-1H-indazole (16d). Yield: $207 \mathrm{mg}(0.87 \mathrm{mmol}, 87 \%)$ as a yellow oil; IR: 2845, $1517 \mathrm{~cm}^{-1} ;{ }^{1} \mathrm{H}-\mathrm{NMR}\left(\mathrm{CDCl}_{3}\right): \delta 7.72(\mathrm{~d}, J=8.1 \mathrm{~Hz}, 1 \mathrm{H}), 7.60(\mathrm{~d}, J=7.7 \mathrm{~Hz}, 1 \mathrm{H}), 7.59(\mathrm{~d}, J=8.9 \mathrm{~Hz}, 2 \mathrm{H})$, $7.39(\mathrm{t}, J=7.4 \mathrm{~Hz}, 1 \mathrm{H}), 7.18(\mathrm{t}, J=7.5 \mathrm{~Hz}, 1 \mathrm{H}), 7.04(\mathrm{~d}, J=8.9 \mathrm{~Hz}, 2 \mathrm{H}), 3.87(\mathrm{~s}, 3 \mathrm{H}), 2.65(\mathrm{~s}, 3 \mathrm{H})$; ${ }^{13} \mathrm{C}-\mathrm{NMR}\left(\mathrm{CDCl}_{3}\right): \delta 158.1,143.8,139.7,133.5,126.9,124.4,124.3,120.50,120.47,114.6,110.1,56.6,11.9$; MS: $m / z 238\left(\mathrm{M}^{+}\right)$, calculated $m / z$ 238.11. Anal. Calcd. for $\mathrm{C}_{15} \mathrm{H}_{14} \mathrm{~N}_{2} \mathrm{O}: \mathrm{C}, 75.61 ; \mathrm{H}, 5.92 ; \mathrm{N}, 11.76$. Found: C, 75.35; H, 6.18; N, 11.25.

4-(3-Methyl-1H-indazol-1-yl)benzonitrile (16k). Yield: $198 \mathrm{mg}(0.85 \mathrm{mmol}, 85 \%)$ as tan solid, m.p. $\quad 124-126{ }^{\circ} \mathrm{C}$; IR: 2224, 1604, $1517 \mathrm{~cm}^{-1}$; ${ }^{1} \mathrm{H}-\mathrm{NMR}\left(\mathrm{CDCl}_{3}\right): \delta 7.91(\mathrm{~d}, J=8.6 \mathrm{~Hz}, 2 \mathrm{H})$, $7.80(\mathrm{~d}, J=8.6 \mathrm{~Hz}, 2 \mathrm{H}), 7.78(\mathrm{~d}, J=8.4 \mathrm{~Hz}, 1 \mathrm{H}), 7.75(\mathrm{~d}, J=7.9 \mathrm{~Hz}, 1 \mathrm{H}), 7.50(\mathrm{t}, J=8.3 \mathrm{~Hz}, 1 \mathrm{H})$, $7.28(\mathrm{t}, J=7.4 \mathrm{~Hz}, 1 \mathrm{H}), 2.65(\mathrm{~s}, 3 \mathrm{H}) ;{ }^{13} \mathrm{C}-\mathrm{NMR}\left(\mathrm{CDCl}_{3}\right): \delta 146.1,143.9,139.1,133.5,128.0,125.9,121.9$, 121.3, 121.1, 118.7, 110.5, 108.5, 12.0; MS: $m / z 233\left(\mathrm{M}^{+}\right)$, calculated $m / z$ 233.10. Anal. Calcd. for $\mathrm{C}_{15} \mathrm{H}_{11} \mathrm{~N}_{3}$ : C, 77.23; H, 4.75; N, 18.01. Found: C, 77.35; $\mathrm{H}, 4.31 ; \mathrm{N}, 18.32$.

1-Phenyl-1H-indazole (17a). Yield: $149 \mathrm{mg}(0.77 \mathrm{mmol}, 77 \%)$ as off-white solid, m.p. $77-78{ }^{\circ} \mathrm{C}$ (lit [18] m.p. $\left.76-78{ }^{\circ} \mathrm{C}\right)$; IR: 1595, $1500 \mathrm{~cm}^{-1} ;{ }^{1} \mathrm{H}-\mathrm{NMR}\left(\mathrm{CDCl}_{3}\right): \delta 8.21(\mathrm{~s}, 1 \mathrm{H}), 7.81(\mathrm{~d}, J=8.1 \mathrm{~Hz}, 1 \mathrm{H})$, 
$7.77(\mathrm{~d}, J=8.4 \mathrm{~Hz}, 1 \mathrm{H}), 7.75(\mathrm{~d}, J=8.7 \mathrm{~Hz}, 2 \mathrm{H}), 7.54(\mathrm{t}, J=8.4 \mathrm{~Hz}, 2 \mathrm{H}), 7.43(\mathrm{t}, J=8.3 \mathrm{~Hz}, 1 \mathrm{H})$, $7.37(\mathrm{t}, J=8.3 \mathrm{~Hz}, 1 \mathrm{H}), 7.23(\mathrm{t}, J=8.1 \mathrm{~Hz}, 1 \mathrm{H}) ;{ }^{13} \mathrm{C}-\mathrm{NMR}\left(\mathrm{CDCl}_{3}\right): \delta 140.2,138.8,135.4,129.5,127.1,126.6$, 125.3, 122.8, 121.5, 121.3, 110.4; MS: $m / z 194\left(\mathrm{M}^{+}\right)$, calculated $m / z$ 194.08. Anal. Calcd. for $\mathrm{C}_{13} \mathrm{H}_{10} \mathrm{~N}_{2}$ : C, 80.39; H, 5.19; N, 14.42. Found: C, 80.15; H, 5.31; N, 14.69 .

1-(4-Methoxyphenyl)-1H-indazole (17d). Yield: $177 \mathrm{mg}(0.79 \mathrm{mmol}, 79 \%)$ as a yellow oil; IR: 2836, $1515 \mathrm{~cm}^{-1} ;{ }^{1} \mathrm{H}-\mathrm{NMR}\left(\mathrm{CDCl}_{3}\right): \delta 8.17(\mathrm{~s}, 1 \mathrm{H}), 7.79(\mathrm{~d}, J=8.1 \mathrm{~Hz}, 1 \mathrm{H}), 7.65(\mathrm{~d}, J=8.5 \mathrm{~Hz}, 1 \mathrm{H})$, $7.62(\mathrm{~d}, J=8.9 \mathrm{~Hz}, 2 \mathrm{H}), 7.41(\mathrm{t}, J=8.3 \mathrm{~Hz}, 1 \mathrm{H}), 7.21(\mathrm{t}, J=8.0 \mathrm{~Hz}, 1 \mathrm{H}), 7.06(\mathrm{~d}, J=8.9 \mathrm{~Hz}, 2 \mathrm{H})$, $3.88(\mathrm{~s}, 3 \mathrm{H}) ;{ }^{13} \mathrm{C}-\mathrm{NMR}\left(\mathrm{CDCl}_{3}\right): \delta 158.4,139.0,134.8,133.4,126.9,124.9,124.5,121.23,121.21,114.6$, 110.2, 55.6; MS: $m / z 224\left(\mathrm{M}^{+}\right)$, calculated $m / z$ 224.09. Anal. Calcd. for $\mathrm{C}_{14} \mathrm{H}_{12} \mathrm{~N}_{2} \mathrm{O}: \mathrm{C}, 74.98 ; \mathrm{H}, 5.39$; $\mathrm{N}, 12.49$. Found: $\mathrm{C}, 74.63 ; \mathrm{H}, 5.52 ; \mathrm{N}, 12.63$.

4-(1H-Indazol-1-yl)benzonitrile (17k). Yield: $164 \mathrm{mg}(0.75 \mathrm{mmol}, 75 \%)$ as white solid, m.p. $104-106{ }^{\circ} \mathrm{C}$ (lit m.p. [18] 103-106 $\left.{ }^{\circ} \mathrm{C}\right)$; IR: 2225, 1604, $1510 \mathrm{~cm}^{-1} ;{ }^{1} \mathrm{H}-\mathrm{NMR}\left(\mathrm{CDCl}_{3}\right): \delta 8.26(\mathrm{~s}, 1 \mathrm{H})$, $7.94(\mathrm{~d}, J=8.6 \mathrm{~Hz}, 2 \mathrm{H}), 7.84(\mathrm{~d}, J=8.6 \mathrm{~Hz}, 2 \mathrm{H}), 7.86-7.81($ complex, $2 \mathrm{H}), 7.51(\mathrm{t}, J=8.4 \mathrm{~Hz}, 1 \mathrm{H})$, $7.31(\mathrm{t}, J=8.0 \mathrm{~Hz}, 1 \mathrm{H}) ;{ }^{13} \mathrm{C}-\mathrm{NMR}\left(\mathrm{CDCl}_{3}\right): \delta 143.8,138.5,137.3,133.6,128.1,126.1,122.5,121.9,121.8$, 118.5, 110.4, 109.3; MS: $m / z 219\left(\mathrm{M}^{+}\right)$, calculated $m / z$ 219.08. Anal. Calcd. for $\mathrm{C}_{14} \mathrm{H}_{9} \mathrm{~N}_{3}: \mathrm{C}, 76.70 ; \mathrm{H}$, 4.14; N, 19.17. Found: C, 76.85; H, 4.31; N, 19.32 .

1-Phenyl-1H-pyrazolo[3,4-b]pyridine (18a). Yield: $133 \mathrm{mg}(0.68 \mathrm{mmol}, 68 \%)$ as a white solid, m.p. 52-54 ${ }^{\circ} \mathrm{C}$ (lit [10] 53-55 ${ }^{\circ} \mathrm{C}$ ); IR: 1595, $1499 \mathrm{~cm}^{-1} ;{ }^{1} \mathrm{H}-\mathrm{NMR} \quad\left(\mathrm{CDCl}_{3}\right)$ : $\delta 8.65(\mathrm{dd}, J=4.5,1.7 \mathrm{~Hz}, 1 \mathrm{H}), 8.28(\mathrm{dd}, J=7.7,1.2 \mathrm{~Hz}, 2 \mathrm{H}), 8.21(\mathrm{~s}, 1 \mathrm{H}), 8.14(\mathrm{dd}, J=8.0,1.7 \mathrm{~Hz}, 1 \mathrm{H})$, $7.54(\mathrm{t}, J=7.7 \mathrm{~Hz}, 1 \mathrm{H}), 7.33(\mathrm{t}, J=7.5 \mathrm{~Hz}, 1 \mathrm{H}), 7.22(\mathrm{dd}, J=8.0,4.5 \mathrm{~Hz}, 1 \mathrm{H}) ;{ }^{13} \mathrm{C}-\mathrm{NMR}\left(\mathrm{CDCl}_{3}\right): \delta 150.1$, 149.2, 139.5, 133.8, 130.2, 129.1, 126.1, 121.4, 117.6, 117.2; MS: $\mathrm{m} / \mathrm{z} 195\left(\mathrm{M}^{+}\right)$, calculated $\mathrm{m} / \mathrm{z} 195.08$. Anal. Calcd. for $\mathrm{C}_{12} \mathrm{H}_{9} \mathrm{~N}_{3}$ : C, 73.83; H, 4.65; N, 21.52. Found: C, 73.95; H, 4.87; N, 21.78.

1-(4-Methoxyphenyl)-1H-pyrazolo[3,4-b]pyridine (18d). Yield: $140 \mathrm{mg}(0.62 \mathrm{mmol}, 62 \%)$ as a purple solid, m.p. $204-205{ }^{\circ} \mathrm{C}$; IR: 2833, $1513 \mathrm{~cm}^{-1} ;{ }^{1} \mathrm{H}-\mathrm{NMR}\left(\mathrm{CDCl}_{3}\right): \delta 9.00(\mathrm{dd}, J=8.0,1.7 \mathrm{~Hz}, 1 \mathrm{H})$, $8.70(\mathrm{dd}, J=4.5,1.7 \mathrm{~Hz}, 1 \mathrm{H}), 8.28(\mathrm{~d}, J=9.0 \mathrm{~Hz}, 2 \mathrm{H}), 7.35(\mathrm{dd}, J=8.0,4.5 \mathrm{~Hz}, 1 \mathrm{H}), 7.26(\mathrm{~s}, 1 \mathrm{H})$, $7.14(\mathrm{~d}, J=9.0 \mathrm{~Hz}, 2 \mathrm{H}), 3.91(\mathrm{~s}, 3 \mathrm{H}) ;{ }^{13} \mathrm{C}-\mathrm{NMR}\left(\mathrm{CDCl}_{3}\right): \delta$ 158.0, 150.6, 149.6, 138.7, 132.9, 132.6, 123.0, 118.3, 115.3, 114.4, 55.6; MS: $m / z 225\left(\mathrm{M}^{+}\right)$, calculated $m / z$ 225.09. Anal. Calcd. for $\mathrm{C}_{13} \mathrm{H}_{11} \mathrm{~N}_{3} \mathrm{O}: \mathrm{C}, 69.32$; H, 4.92; N, 18.66. Found: C, 69.68; H, 5.11; N, 19.82.

4-(1H-Pyrazolo[3,4-b]pyridine-1-yl)benzonitrile (18k). Yield: $172 \mathrm{mg}(0.78 \mathrm{mmol}, 78 \%)$ as a white

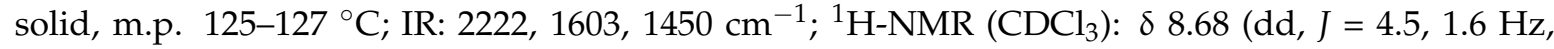
$1 \mathrm{H}), 8.66(\mathrm{~d}, J=8.8 \mathrm{~Hz}, 2 \mathrm{H}), 8.25(\mathrm{~s}, 1 \mathrm{H}), 8.17(\mathrm{dd} . J=8.0,1.6 \mathrm{~Hz}, 1 \mathrm{H}), 7.82(\mathrm{~d}, J=8.8 \mathrm{~Hz}, 2 \mathrm{H})$, $7.30(\mathrm{dd}, J=8.0,4.5 \mathrm{~Hz}, 1 \mathrm{H}) ;{ }^{13} \mathrm{C}-\mathrm{NMR}\left(\mathrm{CDCl}_{3}\right): \delta 150.7,149.4,143.1,135.4,133.2,130.6,120.3,11.89$, 118.4, 117.9, 108.7; MS: $m / z 220\left(\mathrm{M}^{+}\right)$, calculated $m / z$ 220.07. Anal. Calcd. for $\mathrm{C}_{13} \mathrm{H}_{8} \mathrm{~N}_{4}: \mathrm{C}, 70.90$; $\mathrm{H}, 3.66 ; \mathrm{N}, 25.44$. Found: $\mathrm{C}, 71.23 ; \mathrm{H}, 3.82 ; \mathrm{N}, 25.67$.

\section{Conclusions}

We have developed and optimized one and two-pot syntheses of 1-aryl-5-nitro- $1 H$-indazoles from 2'-fluoro-5'-nitroacetophenone (5) and 2-fluoro-5-nitrobenzaldehyde (6). The one-pot conversion of 5 to 11 (a domino reaction) and 6 to 12 (a consecutive reaction) proceeded in yields (70-96\%) that surpassed those of the two-pot sequence. We have also developed a general one-pot approach to 1 -aryl- $1 \mathrm{H}$-indazoles that is not limited by the substitution patterns normally required for the $\mathrm{S}_{\mathrm{N}} \mathrm{Ar}$ ring closure. Treatment of 2'-bromoacetophenone (13), 2-bromobenzaldehyde (14) and 2-chloronicotinaldehyde (15) with $\mathrm{ArNHNH}_{2} \cdot \mathrm{HCl}$ and $30 \mathrm{wt} \%$ of powdered $4 \AA$ molecular sieves in DMPU at $90{ }^{\circ} \mathrm{C}$, followed by addition of $\mathrm{CuI}$ and $\mathrm{K}_{2} \mathrm{CO}_{3}$ afforded $1 \mathrm{H}$-indazoles bearing phenyl as well as electron-rich and electron-poor aromatic groups at N1 in $62-87 \%$ yields. The efficiency of the one-pot process provides access to a selection of 1-aryl- $1 \mathrm{H}$-indazoles in a single laboratory operation-one set-up and one work-up-without isolation or purification of intermediates. The methodology 
described herein has streamlined the synthesis of 1-aryl- $1 H$-indazoles, improved the yields, and minimized the waste generated in preparing these systems.

Supplementary Materials: Electronic Supplementary Information (ESI) are available online: http:/ /www.mdpi. com/1420-3049/23/3/674/s1 Tables summarizing optimization experiments, a listing of characterization data for the intermediate arylhydrazones, and ${ }^{1} \mathrm{H}$ - and ${ }^{13} \mathrm{C}-\mathrm{NMR}$ spectra for all compounds, are available online.

Acknowledgments: The authors wish to acknowledge support from the NSF (BIR-9512269), the Oklahoma State Regents for Higher Education, the W. M. Keck Foundation, and Conoco, Inc. for establishing the Oklahoma State-wide NMR facility. The authors are also grateful to the OSU College of Arts and Sciences for funds that allowed the Chemistry Department to purchase a new $400 \mathrm{MHz}$ NMR for this facility.

Author Contributions: J.K.A.-G. and K.K.G. did the experimental work and developed the one pot protocols. R.A.B. wrote the paper. All of the authors read and approved the final version of the manuscript before submission.

Conflicts of Interest: The authors declare no conflicts of interest.

\section{References and Notes}

1. Nammalwar, B.; Bunce, R.A. Recent Syntheses of 1,2,3,4-tetrahydroquinolines, 2,3-dihydro-4(1H)- quinolinones and 4(1H)-quinolinones using a domino strategy. Molecules 2014, 19, 204-232. [CrossRef] [PubMed]

2. Bunce, R.A.; Nammalwar, B.; Gnanasekaran, K.K.; Cain, N.R. Isoquinolin-1(2H)-ones and 1,6-naphthyridin5(6H)- ones by an $N$-acylation- $S_{N}$ Ar sequence. Tetrahedron 2014, 70, 838-844. [CrossRef]

3. Gnanasekaran, K.K.; Muddala, N.P.; Bunce, R.A. Pyrazoloquinazolinones and pyrazolo- pyridopyrimidinones by a sequential $N$-acylation- $\mathrm{S}_{\mathrm{N}}$ Ar reaction. Tetrahedron Lett. 2015, 56, 1367-1369. [CrossRef]

4. Gnanasekaran, K.K.; Muddala, N.P.; Bunce, R.A. Benzo[4,5]imidazo[2,1- $b$ ]quinazolin-12-ones and benzo[4,5]imidazo[1,2-a]pyrido[2,3- $d$ ]pyrimidin-5-ones by a sequential $N$-acylation- $S_{N} A r$ reaction. Tetrahedron Lett. 2015, 56, 7180-7183.

5. Palazzo, G.; Corsi, G.; Baiocchi, L.; Silvestrini, B. Synthesis and pharmacological properties of 1-substituted 3-dimethylaminoalkoxy-1H-indazoles. J. Med. Chem. 1966, 9, 38-41. [CrossRef] [PubMed]

6. Bai, M.; Carr, G.; DeOrazio, R.J.; Friedrich, T.D.; Dobritsa, S.; Fitzpatrick, K.; Guzzo, P.R.; Kitchen, D.B.; Lynch, M.A.; Peace, D.; et al. 5-Funtionalized indazoles as glucocorticoid receptor agonists. Bioorg. Med. Chem. Lett. 2010, 20, 3017-3020. [CrossRef] [PubMed]

7. Zhao, C.; Wang, R.; Li, G.; Xue, X.; Sun, C.; Qu, X.; Li, W. Synthesis of indazole based diarylurea derivatives and their antiproliferative activity against tumor cell lines. Bioorg. Med. Chem. Lett. 2013, 23, 1989-1992. [CrossRef] [PubMed]

8. Dessole, G.; Branca, D.; Ferrigno, F.; Kinzel, O.; Muraglia, E.; Palumbi, M.C.; Rowley, M.; Serafini, S.; Steinkühler, C.; Jones, P. Discovery of $N$-[(1-aryl-1H-indazol-5-yl)methyl]amide derivatives as smoothened antagonists for inhibition of the hedgehog pathway. Bioorg. Med. Chem. Lett. 2009, 19, 4191-4195. [CrossRef] [PubMed]

9. Mahindroo, N.; Punchihewa, C.; Fujii, N. Hedgehog-gli pathway inhibitors as anticancer agents. J. Med. Chem. 2009, 52, 3829-3845. [CrossRef] [PubMed]

10. Gao, M.; Liu, X.; Wang, X.; Cai, Q.; Ding, K. Synthesis of 1-aryl-1H-indazoles via a ligand-free copper-catalyzed intramolecular amination reaction. Chin. J. Chem. 2011, 29, 1199-1204. [CrossRef]

11. Esmaeili-Marandi, F.; Saeedi, M.; Mahdavi, M.; Yavari, I.; Foroumadi, A.; Shafiee, A. Potassium tert-butoxide promoted intramolecular amination of 1-aryl-2-(nitrobenzylidene)hydrazines: Efficient synthesis of 1-aryl-1H-indazoles. Synlett 2014, 25, 2605-2608.

12. Lokhande, P.D.; Raheem, A.; Sabale, S.T.; Chabukswar, A.R.; Jagdale, S.C. An efficient synthesis of $1 H$-indazoles. Tetrahedron Lett. 2007, 48, 6890-6892. [CrossRef]

13. Xiong, X.; Jiang, Y.; Ma, D. Assembly of $N, N$-disubstituted hydrazines and 1-aryl-1H-indazoles via copper-catalyzed coupling reactions. Org. Lett. 2012, 14, 2552-2555. [CrossRef] [PubMed]

14. Veerareddy, A.; Gogireddy, S.; Dubey, P.K. Regioselective synthesis of 1-substituted indazole-3carboxylic acids. J. Heterocycl. Chem. 2014, 51, 1311-1321. [CrossRef]

15. Wheeler, R.C.; Baxter, E.; Campbell, I.B.; Macdonald, S.J.F. A General one-step synthesis of substituted indazoles using a flow reactor. Org. Process Res. Dev. 2011, 15, 565-569. [CrossRef]

16. Lu, Y.; Cole, K.P.; Fennell, J.W.; Maloney, T.D.; Mitchell, D.; Subbiah, R.; Ramadas, B. An alternative indazole synthesis for merestinib. Org. Process Res. Dev. 2018, 22. [CrossRef] 
17. Li, P.; Wu, C.; Zhao, J.; Rogness, D.C.; Shi, F. Synthesis of substituted $1 H$-indazoles from arynes and hydrazones. J. Org. Chem. 2012, 77, 3149-3158. [CrossRef] [PubMed]

18. Lebedev, A.Y.; Khartulyari, A.S.; Voskoboynikov, A.Z. Synthesis of 1-aryl-1H-indazoles via a palladiumcatalyzed intramolecular amination of aryl halides. J. Org. Chem. 2005, 70, 596-602. [CrossRef] [PubMed]

19. Xu, P.; Wang, G.; Wu, Z.; Li, S.; Zhu, C. Rh(III)-catalyzed double C-H activation of aldehyde hydrazones: A route for functionalized $1 H$-indazole synthesis. Chem. Sci. 2017, 8, 1303-1308. [CrossRef] [PubMed]

20. Schmidt, A.; Beutler, A.; Snovydovych, B. Recent advances in the chemistry of indazoles. Eur. J. Org. Chem. 2008, 4073-4095. [CrossRef]

21. Gaikwad, D.D.; Chapolikar, A.D.; Devkate, C.G.; Warad, K.D.; Tyade, A.P.; Pawar, R.P.; Domb, A.J. Synthesis of indazole motifs and their medicinal importance: An overview. Eur. J. Med. Chem. 2015, 90, 707-731. [CrossRef] [PubMed]

22. Cankarová, N.; Hlavác, J.; Krchnák, V. Recent synthetic approaches to $1 H$ - and $2 H$-indazoles. A review. Org. Prep. Proced. Int. 2010, 42, 433-465. [CrossRef]

23. Tietze, L.F.; Beifuss, U. Sequential transformations in organic chemistry: A synthetic strategy with a future. Angew. Chem. Int. Ed. 1993, 32, 131-163. [CrossRef]

24. Tietze, L.F. Domino reactions in organic chemistry. Chem. Rev. 1996, 96, 115-136. [CrossRef] [PubMed]

25. Tietze, L.F.; Brasche, G.; Gericke, K.M. Domino Reactions in Organic Synthesis; Wiley-VCH: Weinheim, Germany, 2006.

26. Kim, S.; Yoon, J.-Y. Science of Synthesis Houben-Weyl Methods of Molecular Transformations; Padwa, A., Ed.; Thieme: Stuttgart, Germany, 2004; Volume 27, pp. 671-722.

27. Bordwell, F.G. Equilibrium acidities in dimethyl sulfoxide solution. Acc. Chem. Res. 1988, 21, $456-463$. [CrossRef]

28. Reich, H.J.; Rigby, J.H. Handbook of Reagents for Organic Synthesis: Acidic and Basic Reagents; Wiley: New York, NY, USA, 1999; Volume 4, pp. 296-298.

29. Fieser, M.; Fieser, L.F. Reagents for Organic Synthesis; Wiley: New York, NY, USA, 1975; Volume 5, pp. $552-553$.

30. Gribble, G.W. Indole Ring Synthesis: From Natural Products to Drug Discovery; Wiley: New York, NY, USA, 2016; p. 229.

31. Katritsky, A.R.; Ramsden, C.A.; Joule, J.A.; Zhankin, V.V. Most ring closures to produce 6,5-fused aromatic rings involve reaction of an aryl-bound nucleophile with a side chain electrophilic center. In Handbook of Heterocyclic Chemistry, 3rd ed.; Elsevier: Amsterdam, The Netherlands, 2010.

32. Smith, M.B.; March, J. March's Advanced Organic Chemistry: Reactions, Mechanism and Structure, 6th ed.; Wiley-Interscience: Hoboken, NJ, USA, 2007; p. 495.

33. Lukin, K.; Hsu, M.C.; Fernando, D.; Leanna, M.R. New practical synthesis of indazoles via condensation of $O$-fluorobenzaldehydes and their O-methyloximes with hydrazine. J. Org. Chem. 2006, 71, 8166-8172. [CrossRef] [PubMed]

34. Sagitullina, G.P.; Garkushenko, A.K.; Poendaev, N.V.; Sagitullin, R.S. Simple and efficient synthesis of $1 H$-indazoles. Mendeleev Commun. 2012, 22, 167-168. [CrossRef]

35. This statement is further substantiated by the fact that hydrazones bearing nitro groups at C2 and C4 failed to cyclize under our optimized conditions

36. Anderson, K.W.; Tundel, R.E.; Ikawa, T.; Altman, R.A.; Buchwald, S.L. Monodentate phosphines provide highly active catalysts for Pd-catalyzed $\mathrm{C}-\mathrm{N}$ bond forming reactions of heteroaromatic halides/amines and (H)N-heterocycles. Angew. Chem. Int. Ed. 2006, 45, 6523-6527. [CrossRef] [PubMed]

Sample Availability: Samples of the compounds are not available from the authors or MDPI. 